\title{
PIV analysis of near-wake flow patterns of an ice-accreted bridge cable in low and moderately turbulent wind
}

\author{
Piotr Górski ${ }^{\text {a, * }}$, Stanislav Pospíšil ${ }^{\mathrm{b}}$, Marcin Tatara ${ }^{\mathrm{a}}$, Arsenii Trush ${ }^{\mathrm{b}, \mathrm{c}}$ \\ ${ }^{a}$ Department of Roads and Bridges, Faculty of Civil Engineering and Architecture, Opole University of Technology, Katowicka 48, 45-061, Opole, Poland \\ ${ }^{\mathrm{b}}$ Institute of Theoretical and Applied Mechanics, Academy of Sciences of the Czech Republic, Prosecká 76, 190 00, Prague, Czech Republic \\ ${ }^{\mathrm{c}}$ Department of Steel and Timber Structures, Faculty of Civil Engineering, Czech Technical University, Thákurova 7, 166 29, Prague, Czech Republic
}

\section{A R T I C L E I N F O}

\section{Keywords:}

Bridge cable

Ice accretion

Flow visualization

Particle image velocimetry

Near-wake

Vortex street

Velocity field

Streamline patterns

\begin{abstract}
A B S T R A C T
The study contributes to the explanation of flow behaviour and the basis of phenomena existing during twodimensional airflow around an ice-accreted cylinder, representing the section model of a bridge cable. The geometrical features and flow characteristics of the near-wake flow patterns of the iced cable were investigated within the Reynolds number range of $2.2 \cdot 10^{4}-6.4 \cdot 10^{4}$ in low and moderately turbulent flow. The experimental procedure was conducted to create ice accretion on the cylindrical model. The shape of the ice was registered using a photogrammetry method. For the aerodynamic investigations the ice-accreted model was reproduced at a smaller scale by means of 3D printing. Representative snapshots of the flow field behind the stationary horizontal simulated iced cable were digitally analyzed by the Particle Image Velocimetry technique. The flow visualization provided quantitative data about the velocity and the direction of flow streams within both the near-wake and the sidewise regions of distributed flow around the model. Evidence of the existence of the vortex excitation process was obtained at three principal angles of attack. The characteristics of the vortex street and the location of the flow boundary layer separation points were recognized. The obtained results were compared with results for a smooth cylinder.
\end{abstract}

\section{Introduction}

It is well known that the airflow around a bluff body creates air underpressure at the leeward side of the body, i.e. in the wake, and aerodynamic drag force arises. It is also well known that the character of the flow field in the wake, its geometry, the shape of the so-called shear layer near the surface of the body and the location of the points where these layer separate have a decisive influence on the net wind forces acting on the body. Therefore, recognition of phenomena existing during the airflow around bluff bodies, especially knowledge of the resulting aerodynamic force coefficients and the so-called Strouhal number constitute the basis for mathematical models of the wind loads acting on a structure. In the case of bridge cables with nominally circular crosssections, such phenomena are relatively well understood and their mathematical models are presented in the literature, e.g. (Zdravkovich, 1997; Demartino and Ricciardelli, 2017). There are however still several open questions.

In the case of specific meteorological conditions, e.g. during freezing rain or the accumulation of wet snow, the circular cross-section of bridge cables may change due to ice accretion. This can significantly influence the flow field around the cables, the static and dynamic aerodynamic force acting on them and, consequently, it can lead to a much larger amplitude of cable vibrations than is typically seen on smooth cables without ice. The probability of the ideal meteorological conditions necessary for icing bridge cables to occur is not high. On the basis of meteorological observation, it was assumed that icing would most probably occur at air temperatures from $-11^{\circ} \mathrm{C}$ to $2{ }^{\circ} \mathrm{C}$, with precipitation below $0.1 \mathrm{~mm} / \mathrm{h}$ and at relative humidity equal to or above $95 \%$. For example, Roldsgaard et al. (2013) estimated that these conditions conducive to icing occur simultaneously in a total of about $96 \mathrm{~h}$ per year. They used the data from the vicinity of the Øresund Bridge in Denmark and applied Bayesian Probabilistic Network. Despite the relatively low likelihood of atmospheric icing of bridge cables, there were numerous reported accidents leading to unanticipated bridge closures and traffic restrictions due to cable vibrations resulting from the combination of particular wind conditions and ice accretion. Besides these vibration episodes, many safety problems occurred for bridge users due to the phenomenon of ice shedding as well. For example, a number of cable

\footnotetext{
* Corresponding author.

E-mail address: p.gorski@po.opole.pl (P. Górski).
} 

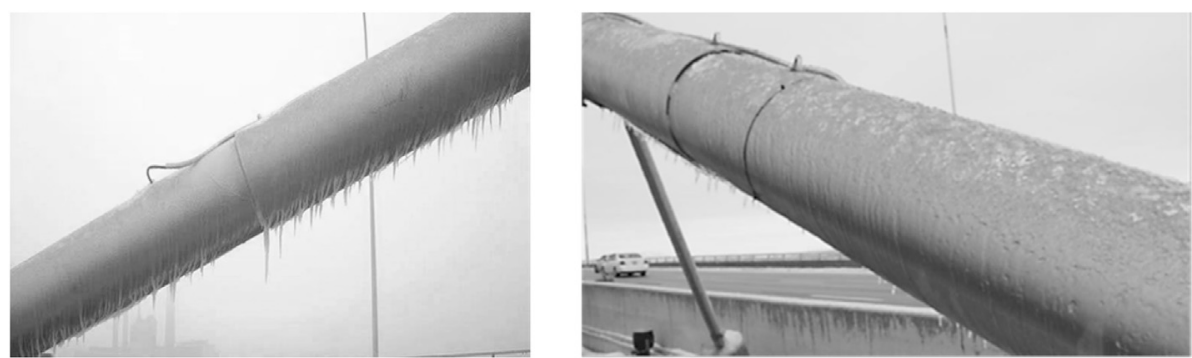

Fig. 1. Example of frozen rivulets and ice accreting on stay cables of the Veteran's Glass City Skyway Bridge in Toledo, Ohio, USA (Nims, 2011).

a)

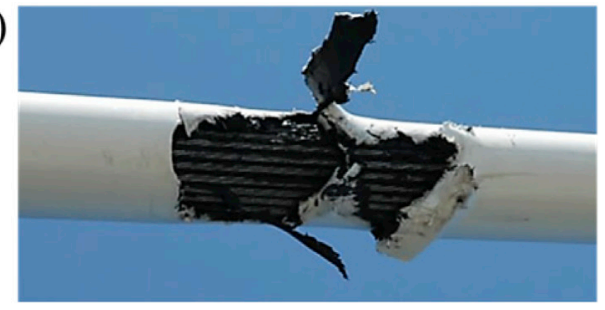

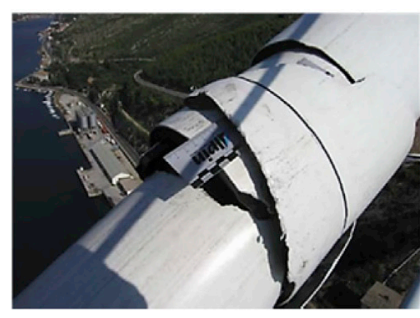

b)

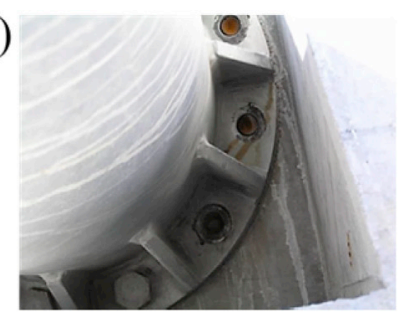

Fig. 2. Example of damage to (a) bridge cable cover sheath and (b) cable anchor resulting from cable icing conditions and wind action at the Dubrovnik Bridge, Croatia (Savor et al., 2006).

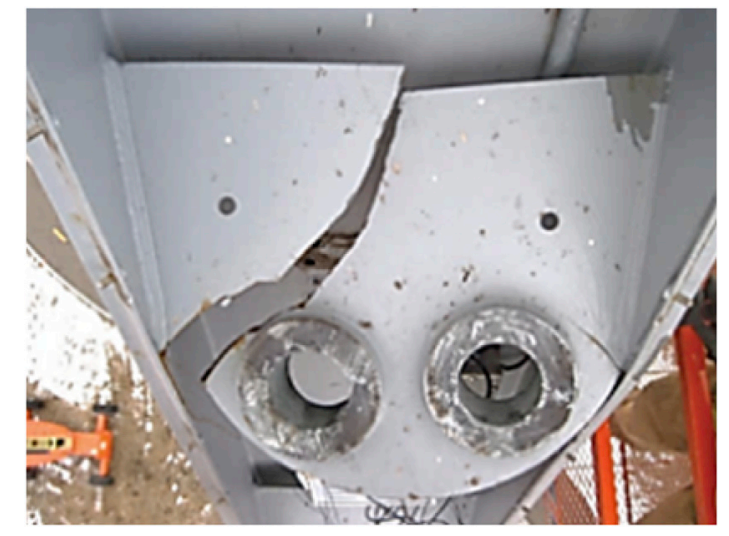

Fig. 3. Failure of cable anchorage due to fatigue cracking as a result of vortex excitation at the Martin Olav Sabo Pedestrian Bridge in Minneapolis, USA (Santosuosso et al., 2012).

icing events are known for the cable-supported Veteran's Glass City Skyway Bridge in Toledo, Ohio, USA (see Fig. 1), which were reported by Nims (2011).

A significant vibration problem which inflicted the Great Belt
Suspension Bridge in Denmark was reported by Gjelstrup et al. (2007). In March 29, 2001, excessive wind-induced vibrations of iced hangers were noted with amplitudes of up to approximately $1.4 \mathrm{~m}$ in the across-wind direction and $1.0 \mathrm{~m}$ in the along-wind direction. The vibrations occurred at a mean wind velocity in the range of $16-18 \mathrm{~m} / \mathrm{s}$. Such large amplitudes were not observed in the case of hangers without ice. This may lead to excessive stress in the cable structure as well as its joints and can cause material fatigue in the cables. Some examples of this type of damage to bridge cables were described by Savor et al. (2006) and are depicted in Fig. 2. Cable stay vibrations of the Dubrovnik Bridge in Croatia with large vibration amplitudes which lead to fatigue problems were registered twice, in March 2005 and May 2006. So far, the exact causes of the excessive cable vibrations have not been explained. However, the hypothesis is that the mechanisms of wind-induced cable vibration phenomena experienced under cable icing conditions could be caused by two alternative phenomena, i.e.: (1) the galloping instability, or (2) a combination of the dynamic wind response of bridge cables to the turbulent wind action and an adverse change to the circular cable cross-section due to the wet snow deposit on the cable cover.

The mechanism of the induced vortices may also cause serious vibration problems for bridge cables. Trush et al. (2017) indicated that the vibration amplitudes caused by vortex excitation of ice-accreted cables can be several times greater than for dry cables. It was found that the
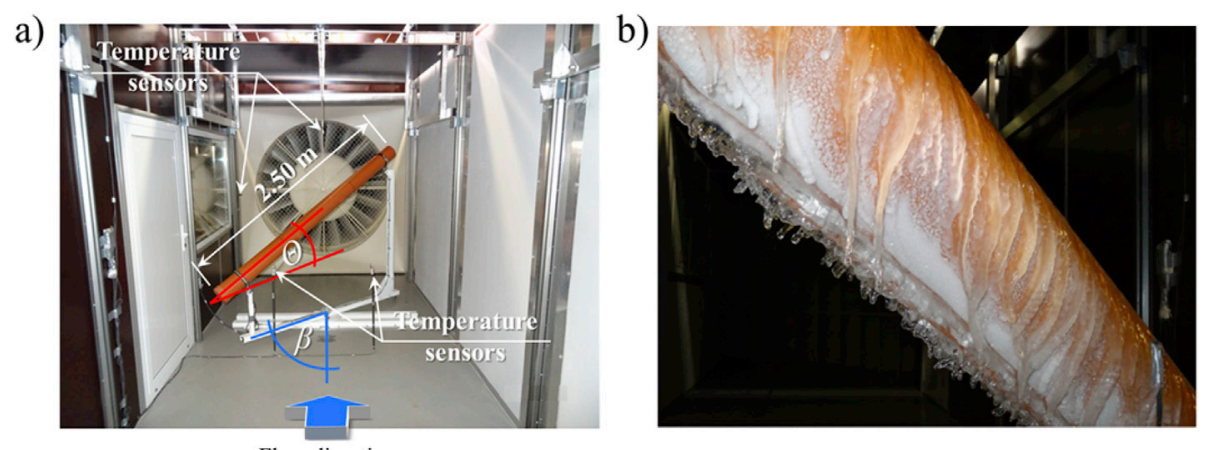

Fig. 4. (a) View of the cable model fixed to a special frame in the climatic chamber of the wind tunnel during the icing process, and (b) view of the final icing effect from below the cable model. 

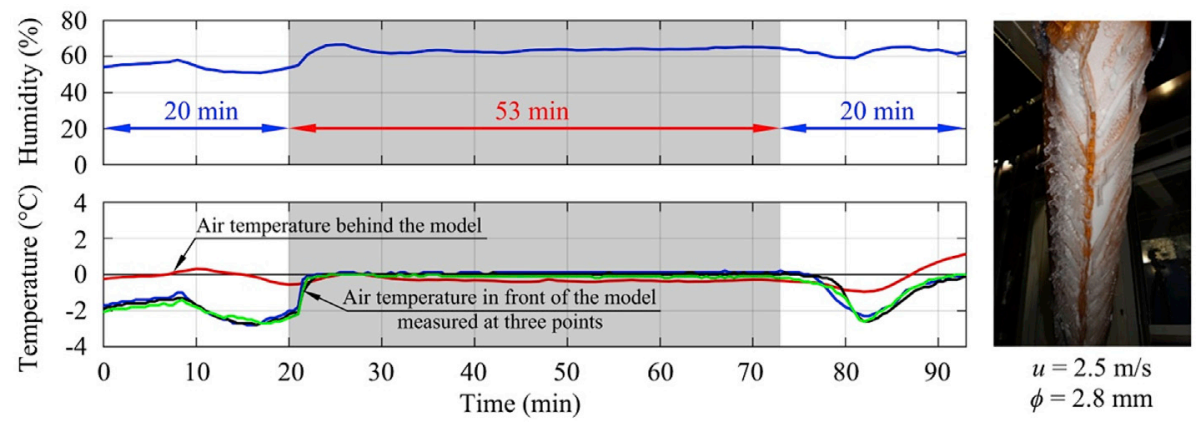

Fig. 5. Air humidity and temperature recorded in the climatic chamber during the icing process of the cable model.

a)

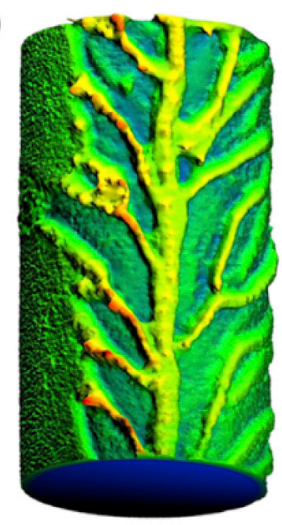

b)

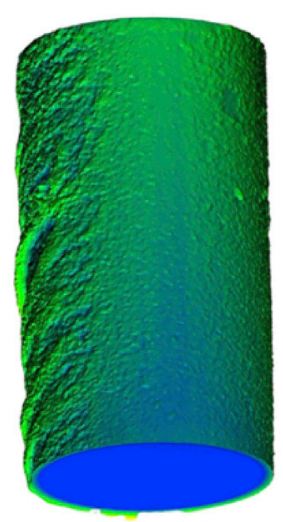

$(\mathrm{mm})$

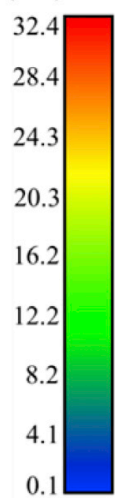

Fig. 6. Three-dimensional numerical model of the ice-accreted cable at a 1:1 scale with determination of the ice layer thickness on (a) the bottom side and (b) the upper side of the model.

highest vibration amplitudes are likely to appear in the case of icing on the leeward cable surface at low turbulent flow. Moreover, assuming a typical cable diameter of $0.16 \mathrm{~m}$, a Strouhal number of 0.18 for the circular cross-section, and a mean wind velocity in the range of $1-30 \mathrm{~m} / \mathrm{s}$, the vortex shedding frequency will exist within the range of about 1-30 Hz. In turn, such wide band frequency vibrations can cause fatigue damage to the anchorage of a cable. An example of such failure of bridge cable anchorage that was a result of vortex excitation at the Martin Olav Sabo Pedestrian Bridge in Minneapolis in USA is presented in Fig. 3.

The vibration problems described above are principally caused by the flexible nature of the bridge cables as well as low structural damping. Currently, various types of cable vibrations are recognized, each associated with a different cause, such as vortex-induced vibrations, turbulentbuffeting vibrations, wake-induced vibrations or galloping instabilities. The understanding and prediction of violent wind-induced vibrations

requires knowledge of the phenomena accompanying airflow around cables with a modified exterior shape.

Some contemporary achievements in this field are presented in the papers (Koss et al., 2012, 2013; Gjelstrup et al., 2012; Demartino et al., 2013a, 2013b, 2015; Koss and Lund, 2013; Demartino and Ricciardelli, 2015; Cao et al., 2018; Li et al., 2016; Górski et al., 2016). The effects of specific conditions on the shape of ice accretion on circular cylinders of $3.81 \mathrm{~cm}$ and $8.9 \mathrm{~cm}$ in diameter, oriented in the vertical and horizontal directions, were studied by Koss et al. (2012). In this study, various climatic conditions were simulated during a series of wind tunnel tests. As results, a qualitative description of the various phases of the accretion process were presented, along with specifications of characteristic ice shape features. In the paper (Gjelstrup et al., 2012) the effect of thin ice accretion on the aerodynamics of a circular cylinder $7 \mathrm{~cm}$ in diameter, representing a vertical bridge hanger, was tested. The aerodynamic coefficients were obtained from both the static and dynamic wind tunnel tests. Then, based on a theoretical approach, the galloping instability was investigated. It was proven that the iced hanger is prone to aerodynamic instability at specific wind incidence angles. Demartino et al. (2015) performed an extensive study on two HDPE cables, vertical and inclined, with diameters of $16 \mathrm{~cm}$. Various climatic conditions simulated in a climatic wind tunnel led to the reproduction of four different ice shapes for the vertical model, and twelve ice shapes for the inclined model. The aerodynamics of the cables were characterized in relation to individual ice shapes. The results confirmed that aerodynamic drag, lift and moment coefficients vary due to ice accretion and provided some explanations of cable vibration in the galloping regime. Later on, these results were used in calculations by Demartino and Ricciardelli (2015), and an analysis of galloping stability was done. Li et al. (2016) conducted a study on six specific ice shapes, namely crescent-shape and D-shape, made of plastic foam. The basic cable was a cylinder with a $12 \mathrm{~cm}$ diameter. Regions with a sudden decrease in the aerodynamic lift coefficient were identified for each model in the wind tunnel tests. Numerical analyses of ice-accreted models were carried out to evaluate potential galloping instability. It was found that the critical wind velocity is much lower than the design a)

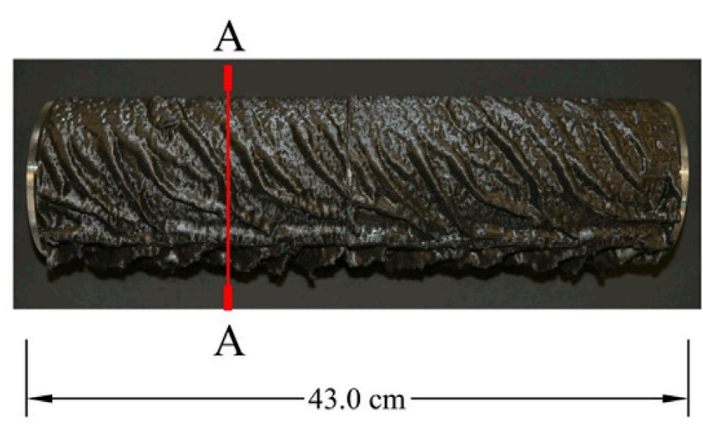

b)

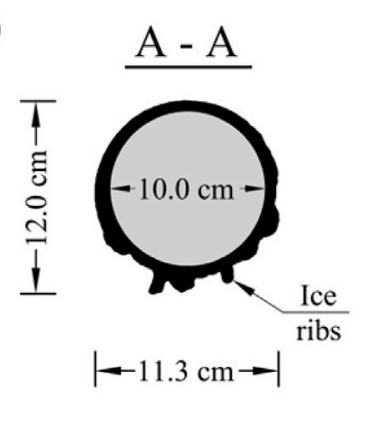

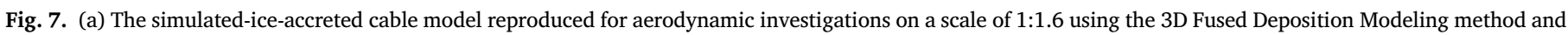
(b) a selected cross-section with the main dimensions. 


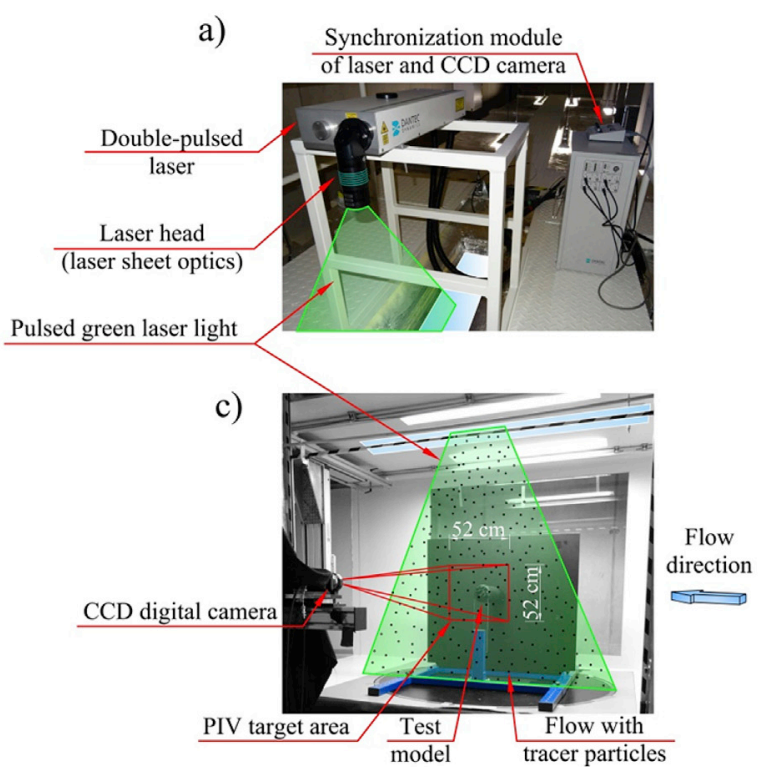

b)
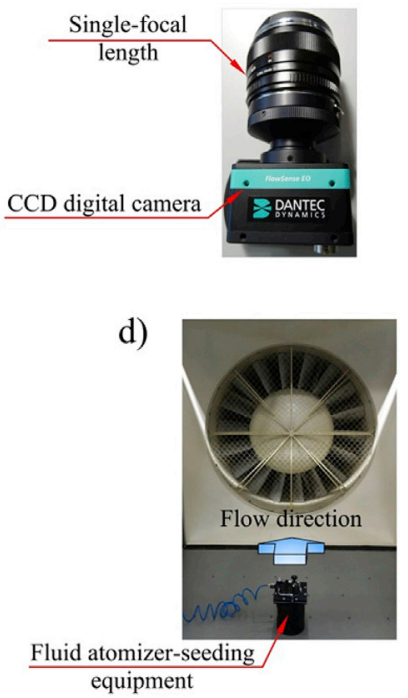

Fig. 8. PIV equipment used for flow visualization around the simulated-ice-accreted cable model: (a) a double-pulsed power laser, (b) a high-rate CCD digital camera, (c) a view of the measuring stand with the PIV target area in the aerodynamic chamber, (d) a high-pressure fluid atomizer for seeding particles.

a)

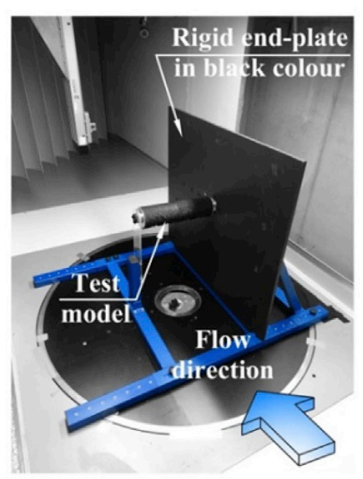

b)

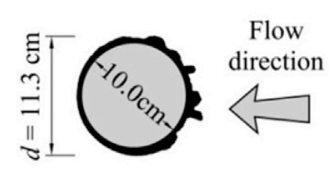

configuration No. 3

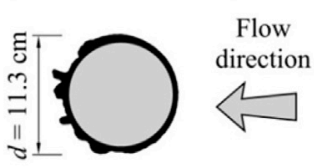

configuration No. 2

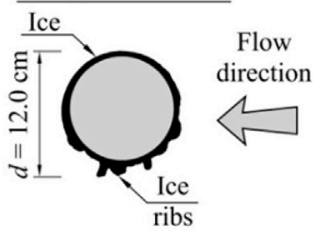

configuration No. $2 \mathrm{~b}$

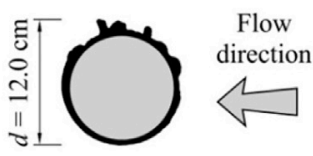

Fig. 9. (a) View of the simulated-ice-accreted model fixed to the frame in the aerodynamic chamber, and (b) model configurations considered in the PIV visualization and their reference dimension $d$ perpendicular to the airflow direction.

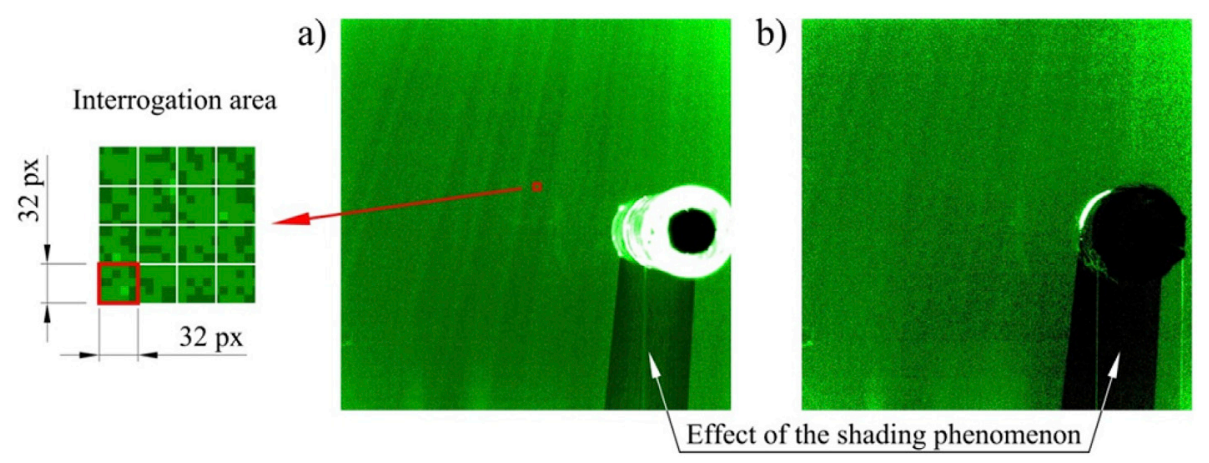

Fig. 10. The effect of the shading phenomenon in the target area of the flow field illuminated by laser light for both (a) the smooth circular cylinder made of Perspex, and (b) the simulated-ice-accreted model made of polylactide plastic.

value.

Cao et al. (2018) experimentally analyzed the wind-induced vibrations of yawed and inclined cable models with two different ice layers, 1.0 and $2.0 \mathrm{~cm}$ in thickness. The entire ice profile was made by applying foam and aluminum foil. Based on the wind tunnel tests and several CFD simulations, the aerodynamic drag and lift coefficients as well as the pressure coefficients of the models were determined. It was stated that the vertical and torsional vibrations of the stay cables increased with the increase of the vertical and yaw angles. The initial experimental investigations on the Strouhal number of ice-accreted bridge cables obtained at low turbulent flow, i.e. with a turbulence intensity of $3 \%$, are presented in the paper (Górski et al., 2016). The tests were conducted on 


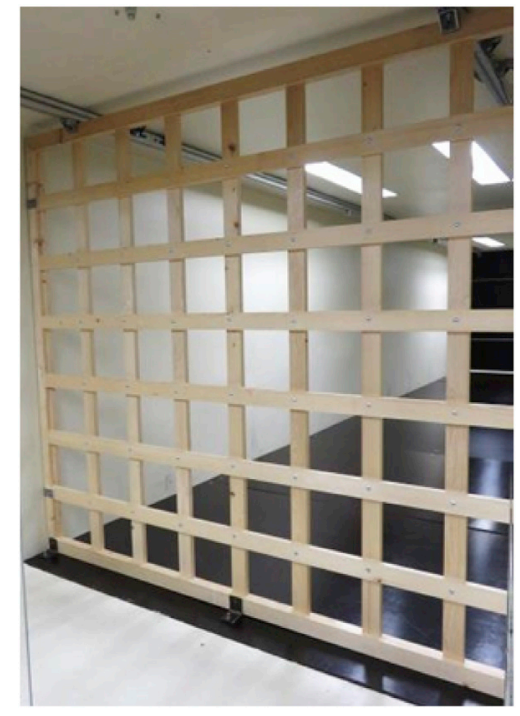

Fig. 11. View of the wooden turbulence grid in the aerodynamic chamber of the wind tunnel.

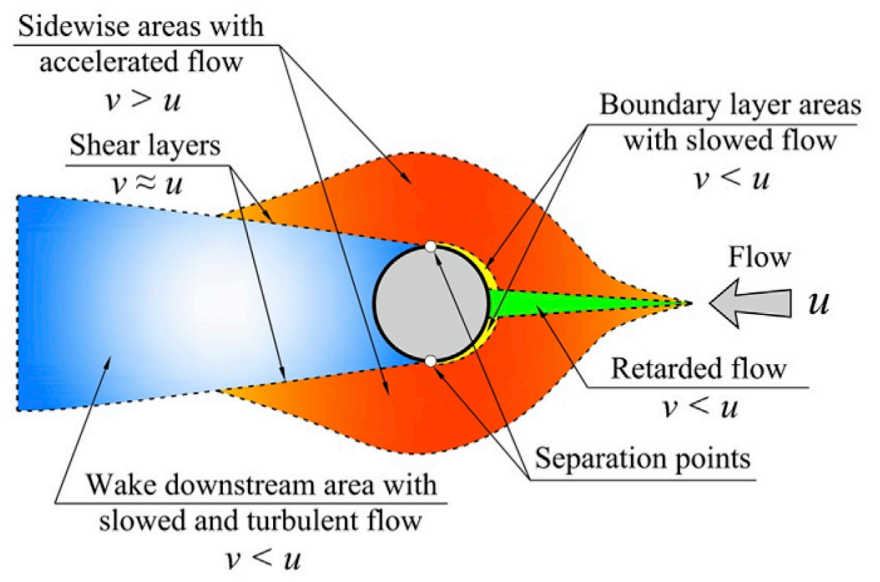

Fig. 12. Schematic division of the flow field around the smooth cylinder into four basic areas (Zdravkovich, 1997; Demartino and Ricciardelli, 2017).

a horizontal model oriented at three principal angles of attack. Based on the test results, the main flow regimes were recognized for three configurations within the studied range of the Reynolds number.

Despite current efforts, knowledge concerning the influence of icing on the aerodynamics of the cables of cable supported bridges is still limited. The majority of existing studies focus on aerodynamic force measurements, thus the phenomena which exist during airflow around ice-accreted cables and the flow characteristics of the near-wake have yet to be thoroughly studied. This is perhaps also due to the fact that the experimental investigations described in some of the above mentioned papers were carried out in wind tunnels only at low streamwise turbulence, i.e. usually at a turbulence intensity of below $1 \%$. However, in many practical applications, the turbulence intensity of the flow field is usually in the range between 10 and $20 \%$. The justification of such a statement can be found in the paper (Zhou et al., 2018), where the mean turbulence intensity with a value of $13.8 \%$ was estimated based on six-year time-average wind data obtained from 2007 to 2012 in the vicinity of the sea-crossing Donghai Bridge, China. It is noteworthy that previous wind tunnel tests conducted by Buresti (1981) or Adachi (1997) have proven that the increased turbulence intensity of the free stream, as well as the increased surface roughness of the cylinder, induce a transition to the so-called critical and trans-critical regimes at lower values of the Reynolds number. Moreover, in turbulent flow conditions, the critical regime can greatly decrease or may even completely disappear. Increased flow turbulence may also produce higher Strouhal number values than in the case of a low turbulent free stream (Cheung and Melbourne, 1983). In some cases (see Trush, 2017) it has even been observed that turbulence suppresses the vibration of a cylinder. Thus, it may be stated that turbulence intensity is a significant parameter having a strong effect on the flow within both the near-wake and the boundary-layer at the cable surface, and, consequently, affects the aerodynamic loading. Therefore, in some respects, the experimental data presented in the above mentioned papers, obtained at low flow turbulence, may suffer from limited practical applications, and cannot be applied unambiguously in practice without corrections. It is therefore necessary to conduct further studies in order to quantify the cable aerodynamics influenced by ice accretion under more realistic conditions.

The primary aim of this paper was to study the geometrical features and the flow characteristics of the near-wake flow patterns of a stationary ice-accreted cable model in low and moderately turbulent flow conditions at various angles of attack. The experimental investigations were carried out in the Climatic Wind Tunnel of the Institute of Theoretical and Applied Mechanics of the Czech Academy of Sciences in Telč. Specific climatic conditions regarding air temperature, wind velocity and precipitation intensity simulating freezing rain or drizzle were selected as the most common conditions for Central Europe. The ice cover on the cable was registered using a photogrammetry method, and a faithful copy of the ice-accreted cable model was reproduced at the smaller scale of 1:1.6 using a 3D printing method.

Optical visualization PIV (Particle Image Velocimetry) of the twodimensional airflow around the simulated ice-accreted cable model and its wake was carried out for the stationary horizontal model positioned perpendicular to the wind direction at free stream velocities within the range of 3.6-8.7 m/s. The corresponding Reynolds number values were within the range of $2.2 \cdot 10^{4}-6.4 \cdot 10^{4}$. Two free stream turbulence intensities were selected to carry out the experiments, $1 \%$ and $17 \%$. The two flow states were defined as the low turbulent and the moderately turbulent flow, respectively. The investigations were performed on the model oriented at three principal angles of attack. The PIV visualization provided quantitative information about the flow structure, velocity and direction of separate flow streams within both the near-wake and the sidewise regions around the model. The location of the separation points of the flow boundary layer, as well as the phenomena existing during two-dimensional airflow, were also recognized. Representative snapshots of the instantaneous flow field around the simulated-ice-accreted cable model were captured and digitally analyzed for three wind velocity cases.

In order to assess the effect of icing on the near-wake characteristics, the results obtained for the simulated-ice-accreted cable were compared with results for a smooth cylinder (reference case). They were in good agreement with the values generally reported in the existing literature for the subcritical Reynolds number range. The results of the investigations, presented in this paper, may help to explain flow behaviour and provide a better understanding of the essential basis of phenomena existing during two-dimensional airflow around an ice-accreted cable. Such studies could constitute the basis for further considerations and case studies of more complex flow, as well as extend knowledge of the physical causes of wind loads acting on bridge cables in icing conditions. This may help understand the current results regarding aerodynamic forces presented in existing literature. In this way, the presented case study can indirectly contribute to an improvement of the reliability of both engineering calculations at the design stage of bridge cables in icing conditions and vibration monitoring of ice-accreted cables excited by wind. According to the authors' knowledge, this paper presents the first instance of a quantitative description of the flow characteristics around an iceaccreted cable model based on PIV flow visualization in both the low and moderately turbulent flow conditions. 
a)

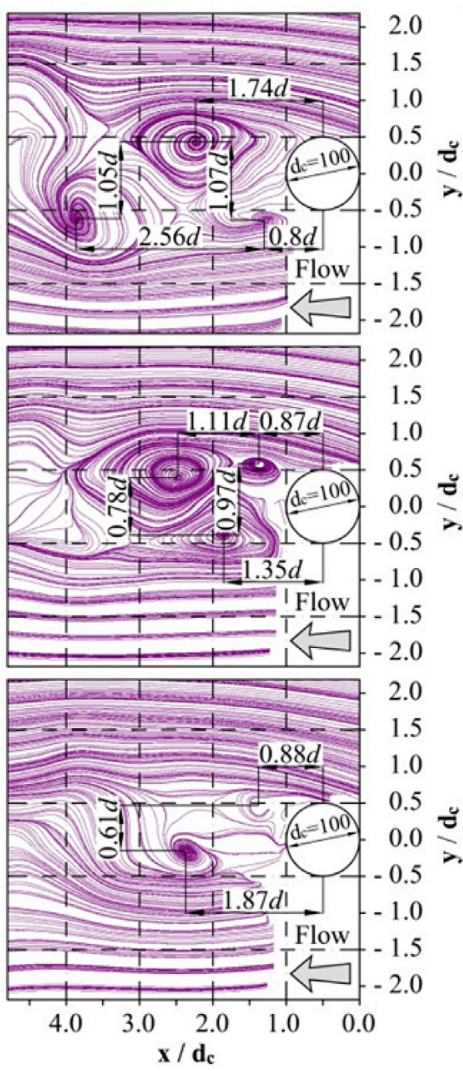

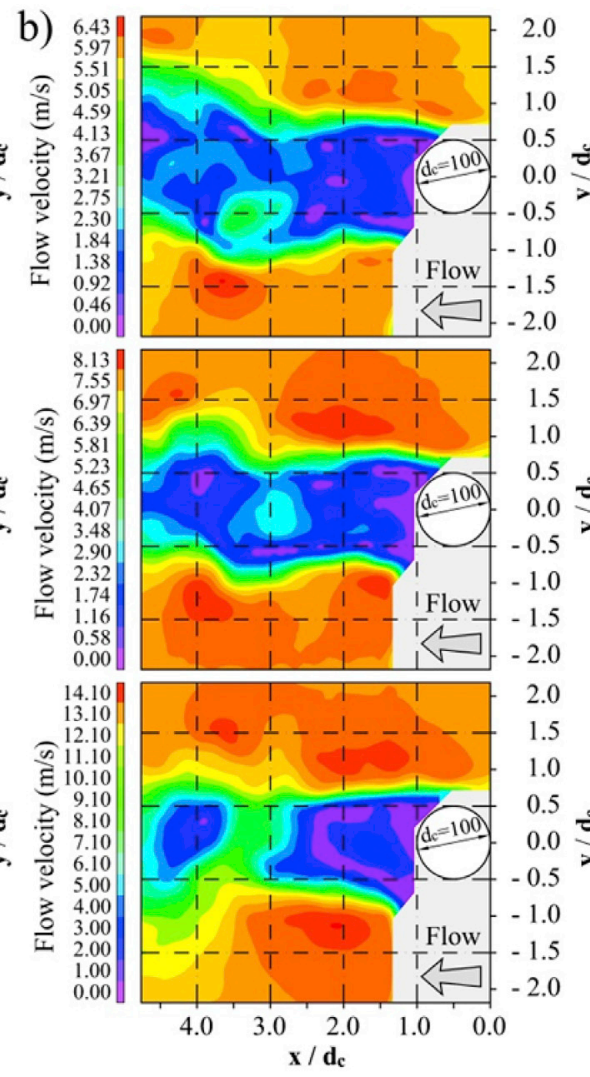

c)

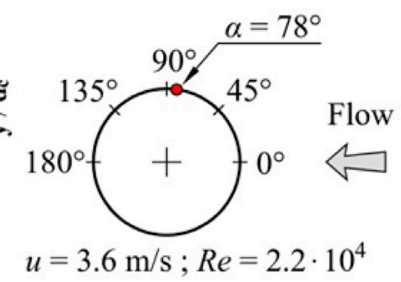

$$
u=3.6 \mathrm{~m} / \mathrm{s} ; \operatorname{Re}=2.2 \cdot 10^{4}
$$

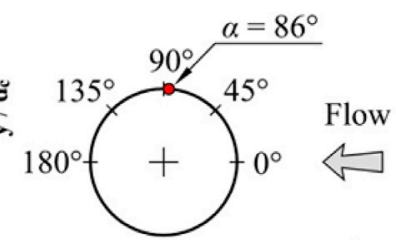

$u=4.9 \mathrm{~m} / \mathrm{s} ; R e=3.0 \cdot 10^{4}$

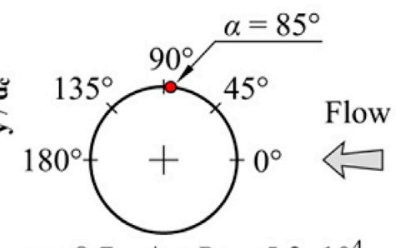

$u=8.7 \mathrm{~m} / \mathrm{s} ; \operatorname{Re}=5.3 \cdot 10^{4}$

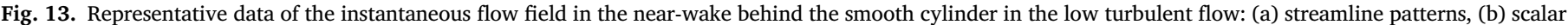
maps of the velocity field, and (c) locations of the flow boundary layer separation point determined at three free stream velocities 3.6 , 4.9 and $8.7 \mathrm{~m} / \mathrm{s}$.

\section{The ice accretion process and preparation of the simulated-ice- accreted cable model for aerodynamic investigations}

An experimental ice accretion process was conducted on a cable section model in the climatic chamber of the wind tunnel in Telč (Kuznetsov et al., 2015). The climatic chamber has a rectangular cross-section $2.5 \mathrm{~m}$ in height and $3.9 \mathrm{~m}$ in width, and is $9.0 \mathrm{~m}$ in length. The $2.5 \mathrm{~m}$ long cable model - a polyvinylchloride pipe with a circular cross-section 16.0 $\mathrm{cm}$ in diameter - was inclined at an angle of $\Theta=30^{\circ}$ to the horizontal plane and yawed at an angle of $\beta=60^{\circ}$ with respect to the mean wind direction. This positioning of the cable model was ensured by mounting it to a special frame (see Fig. 4a).

The icing process was carried out using the wind tunnel cooling system. A typical procedure lasted about $50 \mathrm{~min}$ at an average temperature slightly below $0{ }^{\circ} \mathrm{C}$. The mean free stream velocity $u$ was $2.5 \mathrm{~m} / \mathrm{s}$. Rainy conditions were simulated using rain sprinklers embedded in the laboratory precipitation system in two rows, about $2.0 \mathrm{~m}$ and $2.5 \mathrm{~m}$ ahead of the model. In order to monitor the icing process, an air humidity sensor and a temperature sensor of type Pt1000 were placed inside the chamber. The air temperature was measured at three points located at different heights in front of the model and at one point behind the model (Fig. 4a). Fig. 5 depicts the variation in air humidity and temperature recorded during the experiment. The grey field indicates the icing process proper, i.e. during rainy conditions.

The final ice shape, depicted in Fig. 4b, was obtained for the full-scale bridge cable model. On the underside of the model, the obtained ice shape had characteristic irregular ribs with rounded edges due to frozen rivulets. The relative surface roughness of the ice on this side relative to the cable diameter was estimated at about $18 \%$. On the upper part of the model the ice was nearly circular in shape with a surface roughness of $0.73 \%$. Finally, the outer dimensions of the nonsymmetrical cross-section of the ice-accreted model were $19.2 \mathrm{~cm}$ in height and $18.1 \mathrm{~cm}$ in width.
The final shape of the ice-accreted cable model was registered using a photogrammetry method. Then, a three-dimensional (3D) numerical model of the ice-accreted cable was obtained using numerical image analysis (see Fig. 6). For the aerodynamic investigations, the simulatedice-accreted model, shown in Fig. 7, was reproduced at a smaller scale of 1:1.6 using a 3D printing method commonly known as Fused Deposition Modeling with polylactide plastic as the additive material. Thus, the printed model could be used repeatedly for various aerodynamic tests. The outer dimensions of the cross-section of the resulting cable section were $12.0 \mathrm{~cm}$ in height and $11.3 \mathrm{~cm}$ in width, while its length was $0.43 \mathrm{~m}$. The thickness of the simulated ice layer covering the surface of the model ranged from $7 \mathrm{~mm}$ (on the upper part of the model) to $20 \mathrm{~mm}$ (on the bottom side of the model) - see Fig. 7. More detailed descriptions of the icing process of the cable model in the climatic chamber, the final icing effect and preparation of the simulated-ice-accreted cable model for aerodynamic investigations are presented in the paper (Górski et al., 2016).

\section{PIV flow visualization}

To better understand the nature of the near-wake flow past the iceaccreted cable and to interpret current results of aerodynamic force measurements of cables with simulated ice, the PIV technique was employed. A more detailed description of the principles of the PIV technique can be found in the practical guide (Raffel et al., 2018).

The visualization of the near-wake of the model with simulated ice was made using the following equipment manufactured by Dantec and Litron Lasers (see Fig. 8): (1) a double-pulsed Litron Nd:YAG power laser illuminating green light using laser sheet optics with a double harmonic head $532 \mathrm{~nm}$ and frequency of $47-63 \mathrm{~Hz}$, in which the optics of the laser knife were fixed directly to the head of the laser (Fig. 8a), (2) a high-rate CCD Dantec Dynamics FlowSense EO 4M digital camera equipped with a 
a)

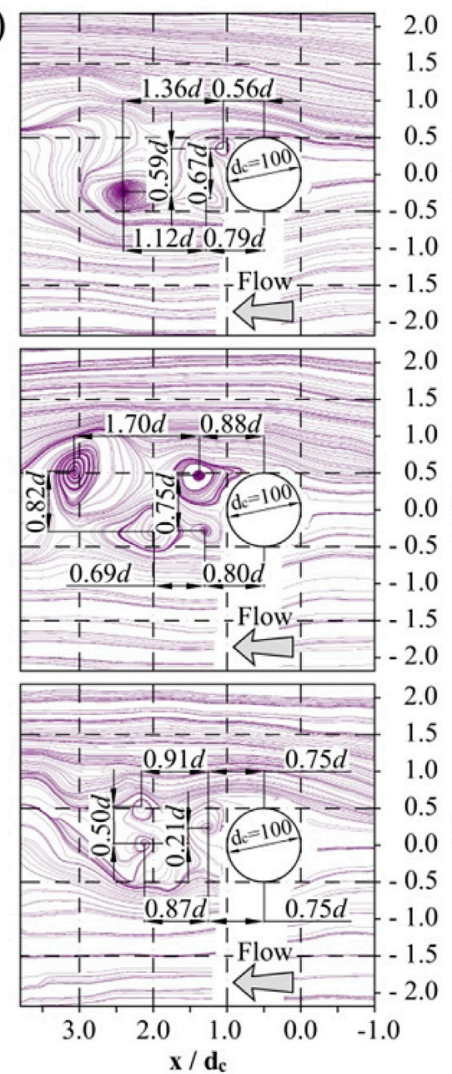

b)
है
$=\frac{2}{0}$
$\frac{0}{0}$
$\frac{3}{4}$

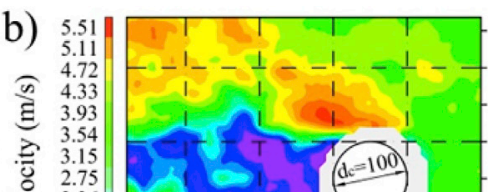

2.0

1.5
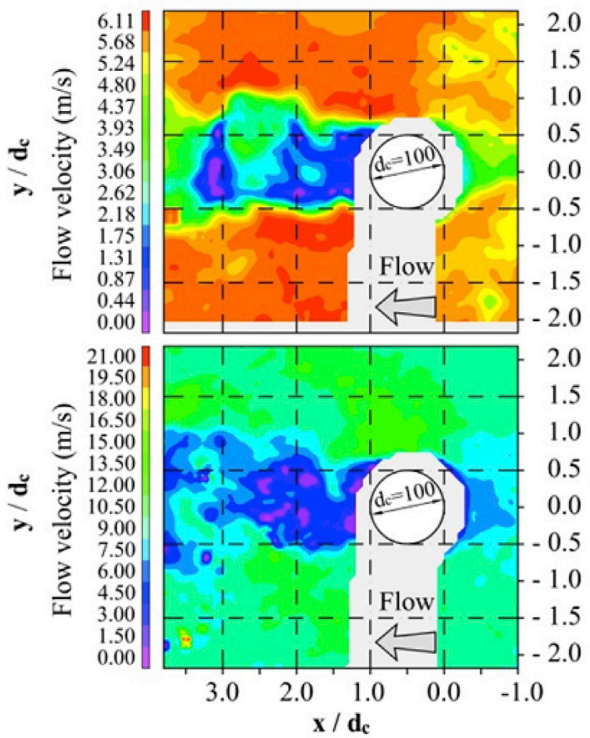

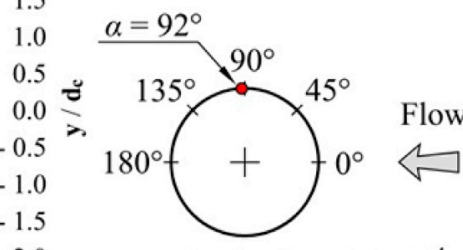

$u=3.6 \mathrm{~m} / \mathrm{s} ; \operatorname{Re}=2.2 \cdot 10^{4}$

c)

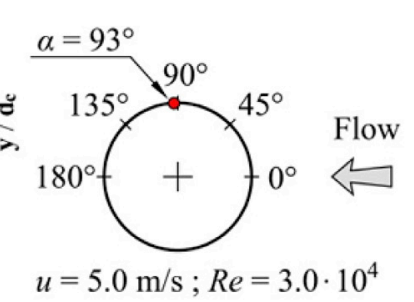

$\alpha=99^{\circ}$

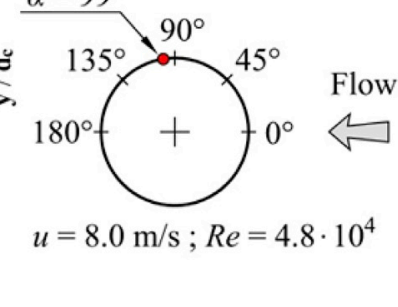

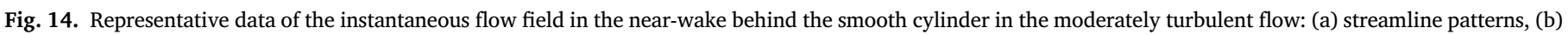
scalar maps of the velocity field, and (c) locations of the flow boundary layer separation point determined at three free stream velocities 3.6 , 5.0 and 8.0 m/s.

single-focal $50 \mathrm{~mm}$ length $\mathrm{f} / 1.4$ lens Zeiss Planar T with high optical performance with a resolution of $2048 \times 2048$ pixels (Fig. 8b), (3) a highpressure fluid atomizer for seeding liquid particles (Fig. 8d), (4) a module for the synchronization of the power laser and the CCD camera (Fig. 8a), (5) the technical software DynamicStudio version 3.31 for image analysis and calculation of the two-component velocity vectors of each particle to compile the wind velocity field into a planar map.

The PIV experiments were conducted in the aerodynamic chamber of the wind tunnel. The dimensions of this rectangular section are $1.8 \mathrm{~m}$ in height, $1.9 \mathrm{~m}$ in width and $11.0 \mathrm{~m}$ in length. The sectional model with simulated ice was fixed horizontally and motionless, perpendicular to the air flow in a special frame at a level of $66 \mathrm{~cm}$ above the floor of the aerodynamic chamber. One side of the frame was equipped with a rigid end-plate, painted black to improve the brightness and the contrast of the PIV technique. A view of the model fixed to the frame is shown in Fig. 9a. The blockage ratio for this experimental setup, computed as the frontal area of the model divided by the total area of a cross-section of the aerodynamic chamber, was about $1.5 \%$ and can thus be disregarded.

To obtain a quantitative velocity field of the flow past the simulatedice-accreted model the standard PIV procedure including the following steps was applied. At first, the entire flow in the closed-circuit wind tunnel was seeded with a mist of oil-based marker particles (Di-EthylHexyl-Sebacate) generated by the high-pressure fluid atomizer, which was placed in front of the ventilator in the climatic chamber (see Fig. 8d). Then, the flow area was illuminated using the high-repetition-rate double-pulsed laser located in the upper part of the aerodynamic chamber and emitting light in the central vertical plane (see Fig. 8a, c). Simultaneously, the square measurement target area of the seeded flow with dimensions $0.52 \times 0.52 \mathrm{~m}$ was recorded digitally by the high-rate CCD camera synchronized with the laser (see Fig. 8 b and c). The optical axis of the camera was pointed at the longitudinal axis of the model. In total, 30 series of conventional PIV velocity field measurements were carried out. and Assuming weak stationarity, averaging was applied to 5-s sequences containing 50 double images (frames) each, i.e. at a sampling rate of $10 \mathrm{~Hz}$. Six sequences made up an ensemble which therefore contained 300 frames. This procedure was chosen due to capacity of the buffer memory of the wind tunnel data acquisition system. The time between each pair of images was $100 \mu \mathrm{s}$. Finally, in order to obtain the velocity data, the captured images were analyzed numerically by the DynamicStudio software (version 3.31) using the adaptive correlation technique available in the program algorithm. A final interrogation area size of $32 \times 32$ pixels (corresponding window size was $8.5 \times 8.5 \mathrm{~mm}$ ) with three iteration steps was set. In the presented case, the experiments were carried out to obtain an overall image of the core vortex concentrations, with a future perspective of using a higher resolution area for more detailed analysis.

The flow visualization was studied for three principal positions (configurations No. 1-3) of the cable model in relation to the mean flow direction, which are presented in Fig. 9b. In this figure the reference dimension $d$, perpendicular to the free stream direction of each model configuration, is also given. It should be noticed that dimension $d$ varies depending on the model orientation relative to the airflow direction and was equal to $d=11.3 \mathrm{~cm}$ for configurations No. 1 and 3 , while $d=12.0 \mathrm{~cm}$ for configurations No. 2 and 2b. For configurations No. 1, 2 and 3 the change in angle of attack was realized by rotating the model around its longitudinal axis every $90^{\circ}$, i.e. a rotation angle of $0^{\circ}, 90^{\circ}$ and $180^{\circ}$, respectively for the three configurations, was assumed.

Due to the non-transparency of the model (reflective in the case of the smooth cylinder made from Perspex) shadows were created beneath the body (see Fig. 10). In the case of the simulated-ice-accreted cable model this deficiency was partially removed by making an additional visualization for configuration No. $2 \mathrm{~b}$.

The tests were carried out both in the low and moderately turbulent flow conditions. The turbulence flow characterization in the test chamber 
a)

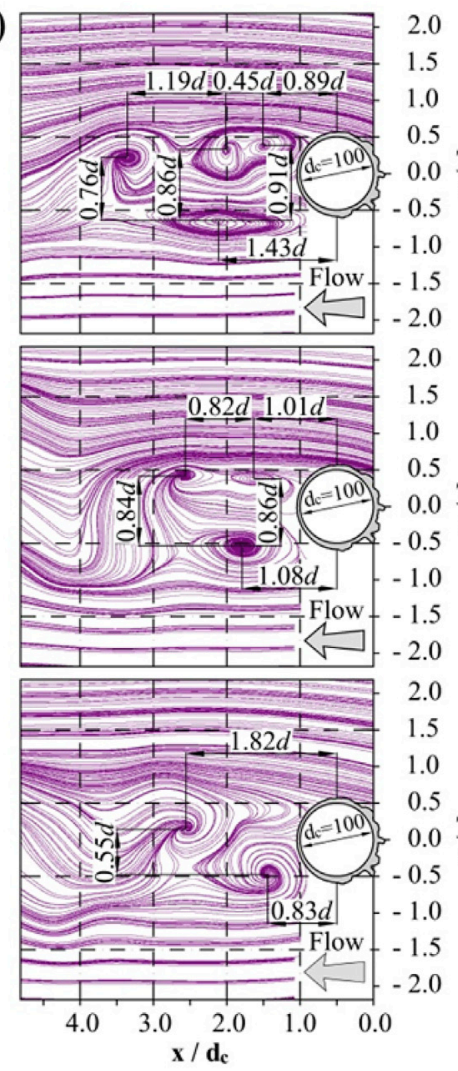

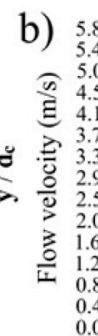

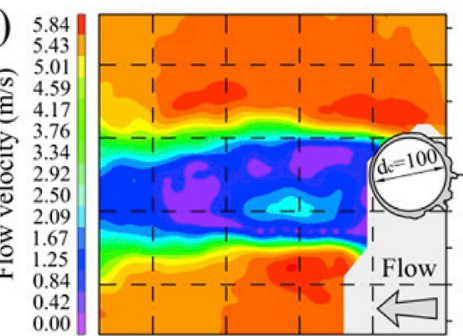

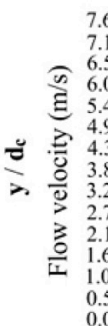

$$
\text { ( }
$$

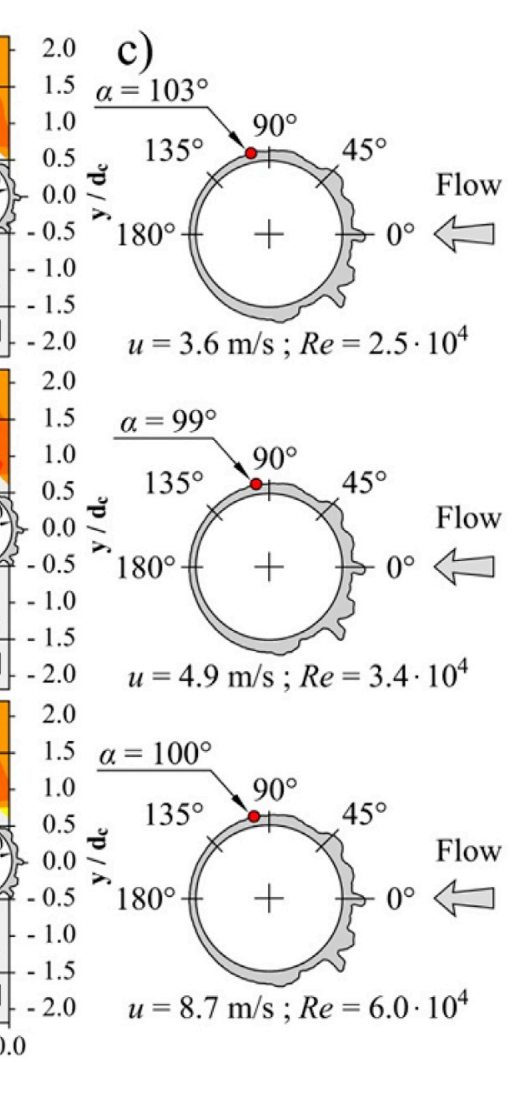

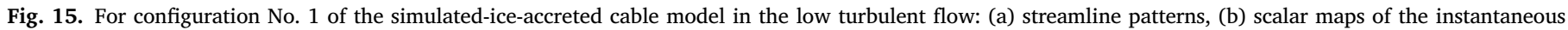
velocity field in the near-wake, and (c) locations of the flow boundary layer separation point determined at three free stream velocities 3.6 , 4.9 and $8.7 \mathrm{~m} / \mathrm{s}$.

was obtained using a CTA (Constant Temperature Anemometry) sensor. At first, the flow in the empty aerodynamic chamber was modelled as the low turbulent flow with the mean turbulence intensity of the order of $1 \%$. In order to produce a turbulent flow a grid of wooden bars was placed in the chamber $2.07 \mathrm{~m}$ upstream of the model. The width and the thickness of the grid bars was $5.5 \mathrm{~cm}$ and $4 \mathrm{~cm}$, respectively, and their spacing was $18 \mathrm{~cm}$. The turbulence intensity at the model position behind the grid was found to be about $17 \%$ and was classified as the moderately turbulent flow. The turbulence grid is depicted in Fig. 11.

During the PIV visualization the free stream velocity $u$ was measured by the Prandtl's tube placed upstream of the model centreline at a distance of $47 \mathrm{~cm}$ and at a level $93 \mathrm{~cm}$ above the floor of the aerodynamic chamber. The tests for the simulated-ice-accreted model were carried out at three mean free stream velocities in the range between $3.6 \mathrm{~m} / \mathrm{s}$ and $8.7 \mathrm{~m} / \mathrm{s}$. The corresponding Reynolds number values based on the reference dimension $d$ were in the range between $2.4 \cdot 10^{4}$ and $6.4 \cdot 10^{4}$. The mean air temperature and the atmospheric pressure inside the aerodynamic section were $25.6^{\circ} \mathrm{C}$ and $962 \mathrm{hPa}$, respectively, for the tests at the low turbulent flow, while for the tests at the moderately turbulent flow these values were $28.3^{\circ} \mathrm{C}$ and $958 \mathrm{hPa}$, respectively.

In order to validate the performance of the PIV visualization, the flow visualization was first performed for a smooth cylinder (made of Perspex) with a diameter of $d_{c}=10.0 \mathrm{~cm}$ and the same length as the simulated-iceaccreted model. During the tests for the smooth cylinder the mean free stream velocity $u$ was also in the range between $3.6 \mathrm{~m} / \mathrm{s}$ and $8.7 \mathrm{~m} / \mathrm{s}$ with the corresponding Reynolds number values in the range from $2.2 \cdot 10^{4}$ to $5.3 \cdot 10^{4}$. It is noteworthy that in the case of a smooth cylinder the studied Reynolds number range was within the subcritical regime in conditions of low flow turbulence (Buresti, 1981). However, as demonstrated in the paper (Cheung and Melbourne, 1983), the studied Reynolds number range for moderate and high flow turbulence corresponds to the transition range from the subcritical to the critical regime. This is due to the interaction of the free stream turbulence with the boundary layer, the free shear layer and the wake, resulting, inter alia, in an earlier transition from laminar to turbulent boundary layer separation over the cylinder surface.

\section{Flow visualization results}

Since the cylinders have special aerodynamic characteristics (Pantazopoulos, 1994; Niemann and Hölscher, 1990), the description of the investigation results obtained for all configurations of the simulated-ice-accreted cable model was referred to the experimental results obtained for a smooth cylinder in the same flow conditions. A schematic view of the disturbed field flow around the circular cylinder consisting four basic areas characterized by the flow velocity denoted as $v$ in given in Fig. 12.

Figs. 13 and 14 depict representative snapshots of the instantaneous flow field in the near-wake behind the smooth cylinder at the three free stream flow velocities $u$ in both turbulent conditions. Since the PIV investigations were conducted for the cable model in a cross-flow, one should be aware that the effect of an axial flow along the cable axis on the vortex shedding existing in the case of an inclined and yawed cable was omitted. In Figs. 13a-14a typical streamline flow patterns indicating the direction of each separate flow as well as vorticity maps are depicted. In these figures the geometrical features of the near-wake behind the cylinder were determined with respect to the reference dimension of $d=10.0 \mathrm{~cm}$. Figs. 13b-14b show the scalar maps of the velocity field in the near-wake colored by the flow velocity magnitude; the cold colors indicate a flow velocity lower than the mean free stream velocity, while the warm colors indicate a flow velocity higher than the mean free stream velocity. The locations of the flow boundary layer separation point are indicated in Figs. 13c-14c. These locations were determined based on the instantaneous flow and were specified by an angle referenced to the 
a)

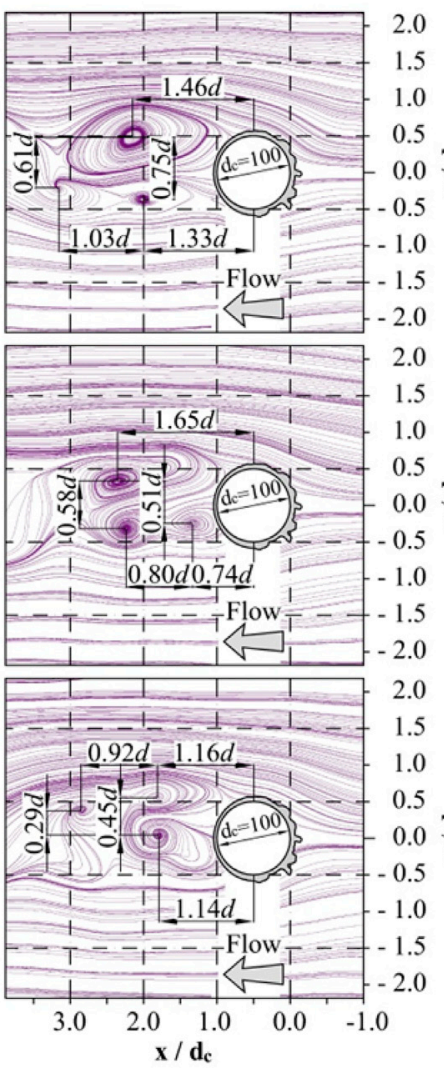

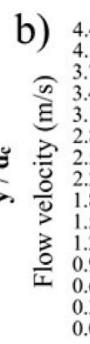
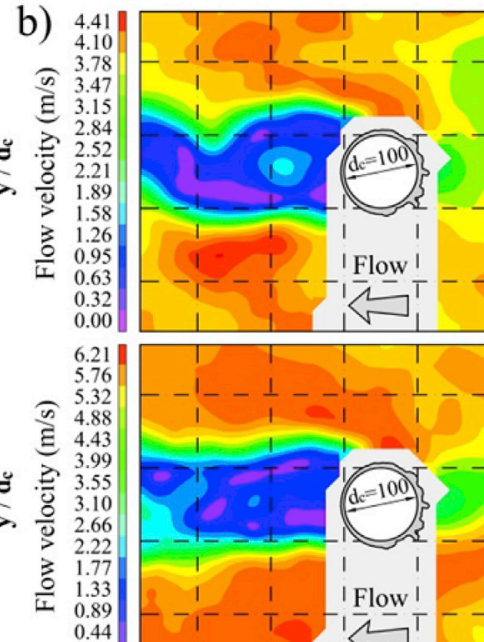

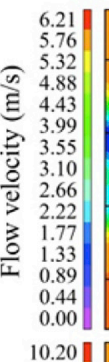
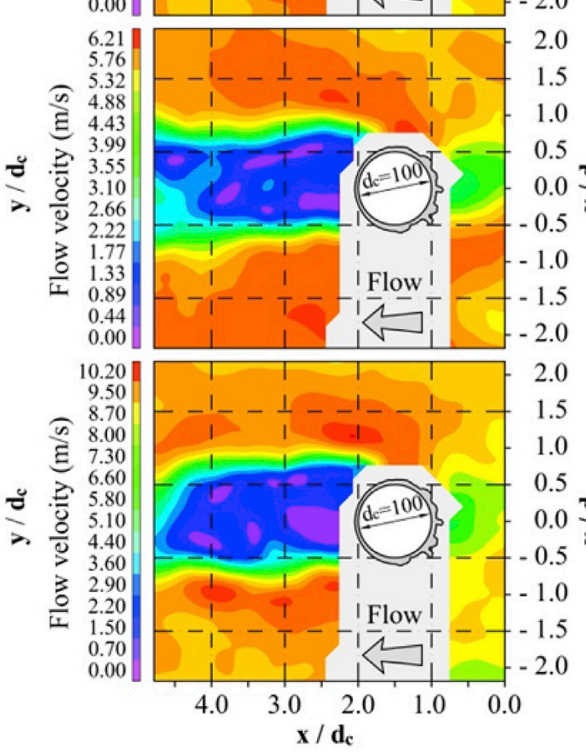

$2.0 \mathrm{c})$

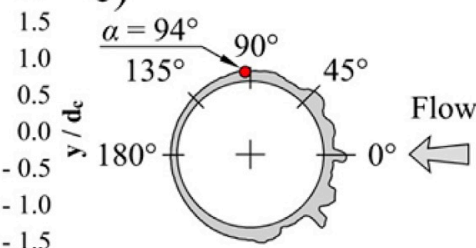

$u=3.6 \mathrm{~m} / \mathrm{s} ; \operatorname{Re}=2.4 \cdot 10^{4}$

0

1.5

1.0

0.5 巳一

$0.5^{>} 180^{\circ}$

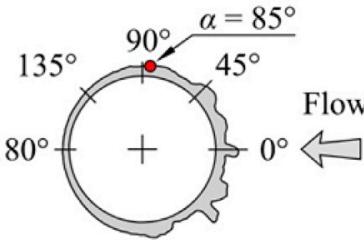

$u=5.0 \mathrm{~m} / \mathrm{s} ; \operatorname{Re}=3.4 \cdot 10^{4}$

$\alpha=92^{\circ}$

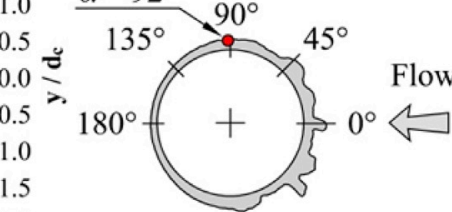

$u=8.0 \mathrm{~m} / \mathrm{s} ; \operatorname{Re}=5.4 \cdot 10^{4}$

Fig. 16. For configuration No. 1 of the simulated-ice-accreted cable model in the moderately turbulent flow: (a) streamline patterns, (b) scalar maps of the instantaneous velocity field in the near-wake, and (c) locations of the flow boundary layer separation point determined at three free stream velocities 3.6, 5.0 and $8.0 \mathrm{~m} / \mathrm{s}$.

location of the stagnation point, denoted as $\alpha$. In all the snapshots the flow direction is considered from right to left. One should be aware that the data obtained in this study is provided with some experimental uncertainty.

As shown in Fig. 13, in the case of the low turbulent flow coming at the cylinder, the wake beyond the cylinder is turbulent and the primary mature vortex pair forming in the wake can be seen clearly. The vortices arise at either side of the cylinder, and a classical Karman vortex street occurs in the wake. The boundary layer attached to the cylinder surface from the windward side is laminar upon the separation points. Two free shear layers forming in the near-wake behind both sides of the cylinder form quasi-stable boundaries between the turbulent wake and both the upper and lower sidewise regions with laminar accelerated flow. Within the two sidewise regions the flow velocity is higher than the mean free stream velocity $u$, reaching a value of about $1.6 u-1.8 u$. Within the nearwake region, the flow velocity is lower than the mean free stream velocity $u$ with a mean value of $0.2 u-0.3 u$. The separation points of the boundary layer, determined with some uncertainties based on an averaging of the 5-s ensembles as described above, takes place at a mean distance of about $78^{\circ}-80^{\circ}$ from the stagnation point, leading to a width of the near-wake, defined as the distance between two shear layers in the cross-stream direction, of approximately $1.5 d$. The cross-stream distance between two primary vortices reached a mean value of about $1.0 d$. The locations of the vortex centers were determined by the position of streamlines with characteristic shapes. The formation length of the two primary mature vortices, defined as the distance between the centers of two consecutive vortices in the same line forming in the streamwise direction, is approximately equal to $2.6 \mathrm{~d}$. Since the drag coefficient is a resultant of aerodynamic forces acting on the body in the along-wind direction, hence, it is to be expected that the wider near-wake leads to higher values for the drag coefficient. Moreover, in the subcritical regimes the vortex shedding frequency in the wake of the cylinder is proportional to the mean free stream velocity, and the vortex Strouhal number is nearly constant at a value of 0.18 .

Comparing the flow structure in the near-wake past the cylinder in the moderately turbulent flow with $17 \%$ turbulence intensity, shown in Fig. 14, with that obtained in the low turbulent flow, depicted in Fig. 13, the weakening of the vortex excitation resulting from the free stream turbulence is clearly visible from the smaller size of the vortices. A similar regularity applies to the formation length of the vortices which decreased from the value $2.6 d$ to $1.7 d$, leading to a higher Strouhal number value than in the case of the smooth flow. This statement is in agreement with findings reported by (Cheung and Melbourne, 1983). One should be aware that the vortex shedding mode and the vorticity concentration affects the fluctuating component of the aerodynamic force in the cross-wind direction, consequently affecting the dynamic cross-wind response of the cylinder. The separation point of the boundary layer is shifted downstream to a mean distance of $95^{\circ}-102^{\circ}$ as the flow turbulence increases. Consequently, the cross-stream distance of two primary vortices decreased to the mean value of approximately $0.8 d$, while the width of the near-wake decreased to approximately $1.0 \mathrm{~d}$, leading to a reduction of the mean drag coefficient. In the moderately turbulent flow conditions the free shear layers become more unstable as opposed to the low turbulent flow, which results in a significantly more irregular shape of the shear layers. Within the upper and lower sidewise regions, as might be expected, the flow is more turbulent, with the flow velocity reaching the mean value in the range of $1.1 u-1.5 u$, while within the near-wake region the mean flow velocity was about $0.2 u$. The obtained experimental results described above are in good agreement with generally reported data for the subcritical Reynolds number range of a cylinder (Cheung and Melbourne, 1983).

Figs. 15-22 depict the representative instantaneous flow field 
a)

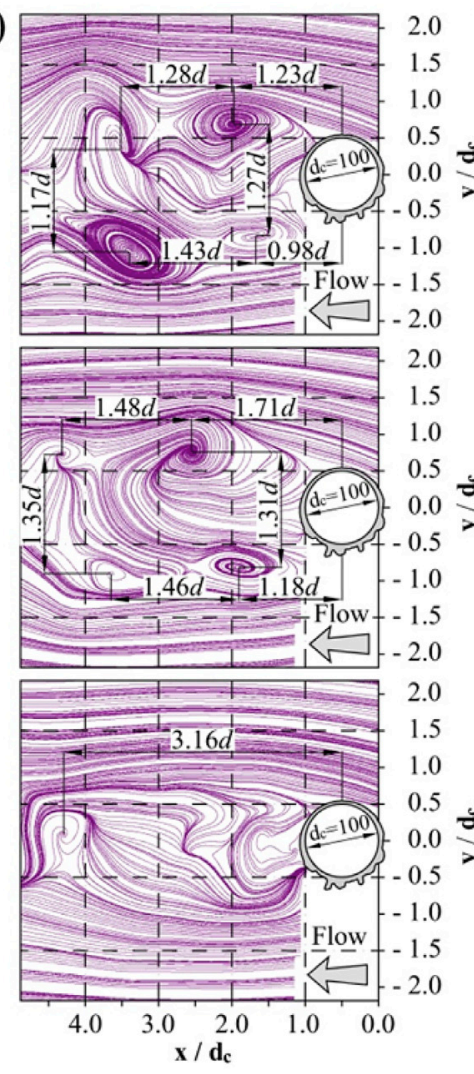

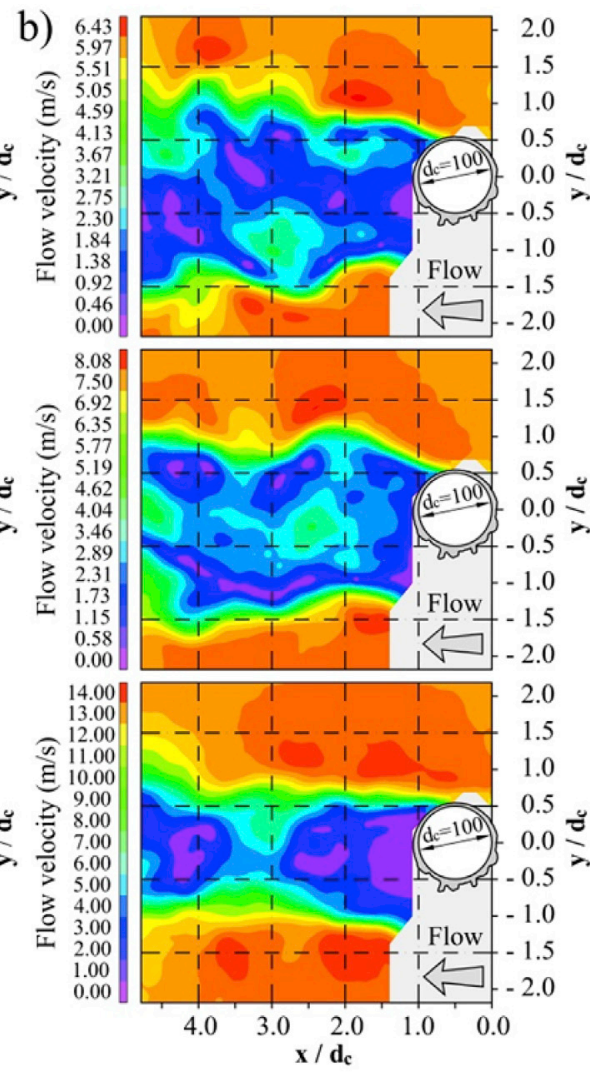

c)

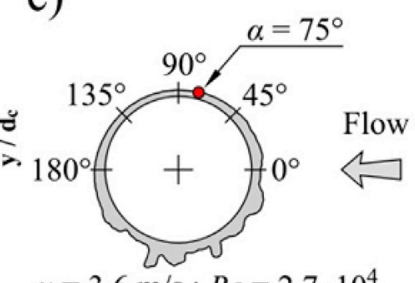

$u=3.6 \mathrm{~m} / \mathrm{s} ; \operatorname{Re}=2.7 \cdot 10^{4}$

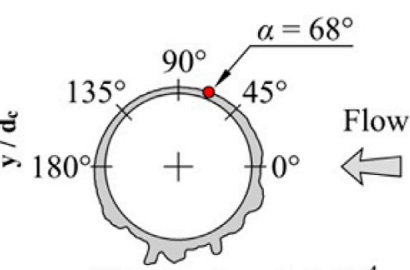

$u=4.9 \mathrm{~m} / \mathrm{s} ; \operatorname{Re}=3.6 \cdot 10^{4}$

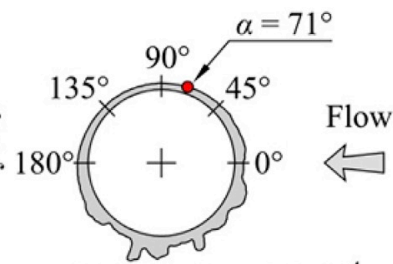

$u=8.7 \mathrm{~m} / \mathrm{s} ; \operatorname{Re}=6.4 \cdot 10^{4}$

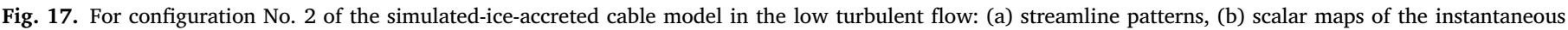
velocity field in the near-wake, and (c) locations of the flow boundary layer separation point determined at three free stream velocities 3.6 , 4.9 and $8.7 \mathrm{~m} / \mathrm{s}$.

snapshots obtained from the PIV visualization of the near-wake behind the simulated-ice-accreted cable for all considered configurations, namely configuration No. $1,2,2 \mathrm{~b}$ and 3 , in the low and moderately turbulent flow at three stream flow velocities $u$ in the range between $3.6 \mathrm{~m} / \mathrm{s}$ and $8.7 \mathrm{~m} / \mathrm{s}$ (the Reynolds number between $2.4 \cdot 10^{4}$ and $6.4 \cdot 10^{4}$ ). In these figures, the streamline patterns and the scalar maps of the velocity field in the near-wake past the model are depicted.

Similar to the flow field around a circular cylinder, the separation region of the boundary layer attached to both the upper and lower areas sidewise of the simulated-ice-accreted cable surface is relatively small, and in practice it is reduced to boundary layer separation points. The instantaneous locations of the points depend on the model configuration, the turbulence intensity and the Reynolds number, and were also denoted in Figs. $15-22 \mathrm{c}$ by the angle $\alpha$. Behind the separation points, the socalled free shear layers are formed. The shear layers are strongly turbulent and depart from the simulated-ice-accreted model with a velocity approximately equal to the free stream velocity $u$. The shear layers separate two flow areas with completely different flow structures. Outside, two sidewise accelerated flows develop. They are usually regular, without vorticity concentration and have quasi-stable streamlines. The flow velocity in this region is much higher than $u$. Another flow is enveloped by the shear layers from either side of the cable model. Between the shear layers, the flow area, i.e. the near-wake, is strongly turbulent and unstable with vortices arising periodically and moving in the streamwise direction behind the model. In this area a region with high turbulence develops with pressure inside it remaining low as the flow separates. It is very interesting that, despite the significant change in the shape of the cable surface caused by the ice accretion in the nearwake of the simulated-ice-accreted model, the phenomenon of vortex excitation can usually be clearly observed having features similar to a circular cylinder. Moreover, this phenomenon occurs for all configurations, i.e. for configuration No. 1, 2 (2b) and 3 in both the low and moderately turbulent flow conditions. However, the free stream turbulence interacts with the free shear layer and the near-wake, having a significant effect on the vorticity concentration and shedding modes.

In the case of configuration No. 1, in the low turbulent flow (see Fig. 15) traversing past the stationary simulated-ice-accreted cable, vortices develop alternately on both the upper and the lower sides of it. This alternating vortex formation behaves quasi-periodically. The oscillating wakes generate staggered rows of vortices with opposite rotation behind the model as in the case of the circular cylinder. However, the vortex street becomes narrower when the cross-stream distance between two primary mature vortices is about $0.8 d$ (the reference dimension is equal to $d=11.3 \mathrm{~cm}$ ) and the width of the near-wake about $1.2 d$. The mean flow velocity within the sidewise regions was $1.4 u-1.6 u$, while in the near-wake region about $0.3 u$. The formation length of the vortices is considerably shorter than that of the smooth cylinder, namely $1.2 d$, with mean separation at around $96^{\circ}-101^{\circ}$.

Apart from the low turbulent flow, in the case of configuration No. 1 in the moderately turbulent flow conditions (see Fig. 16), two single simultaneous vortices per one shedding cycle are fed into the downstream on both sides of the simulated-ice-accreted model. These double vortices are non-stationary, and they move downstream of the wake past the model. In these conditions, mean separation of the boundary layer takes place at $82^{\circ}-102^{\circ}$, which is reflected in the narrowest near-wake having a width of about $1.1 d$ and a distance between vortices in the cross-stream direction of $0.6 d-0.7 d$. The formation length was approximately $0.9 d$, however the flow had not enough time to generate clearly repeatable vortex patterns within the target area. The mean flow velocity in the sidewise regions was $1.1 u-1.2 u$, while in the near-wake it was $0.2 u$ $0.3 u$.

Similar to the cylinder, for configuration No. 2 or $2 b$ (see Figs. 17-20) distinct vortex pairs per one shedding cycle form alternately on both sides of the model. In the case of the low turbulent flow (Figs. 17 and 19) 
a)

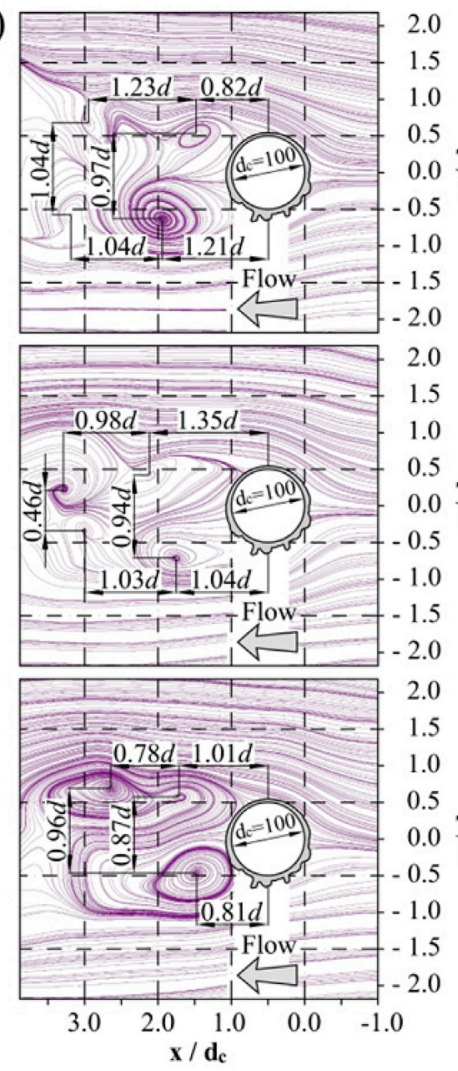

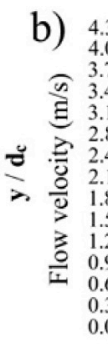
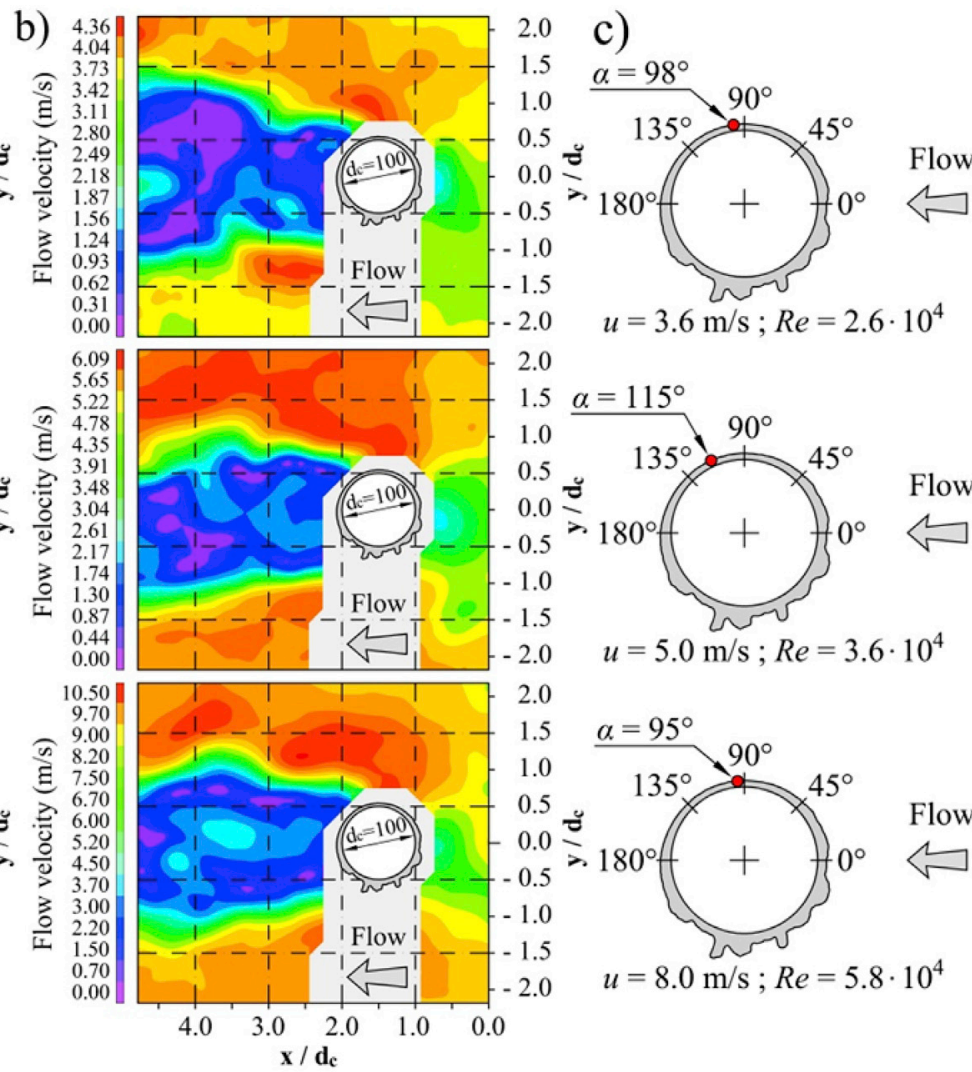

2.0

$u=3.6 \mathrm{~m} / \mathrm{s} ; \operatorname{Re}=2.6 \cdot 10^{4}$

2.0

$1.5 \alpha=115^{\circ}$

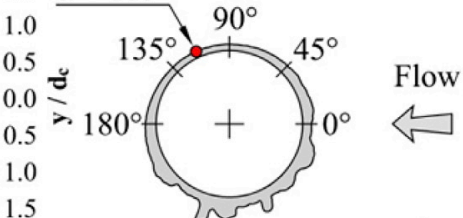

$u=5.0 \mathrm{~m} / \mathrm{s} ; \operatorname{Re}=3.6 \cdot 10^{4}$

2.0

$1.5 \alpha=95^{\circ}$

1.0

$0.5 \cong 0.0$

$-0.5$

$-1.0$

$-2.0 \quad u=8.0 \mathrm{~m} / \mathrm{s} ; \operatorname{Re}=5.8 \cdot 10^{4}$

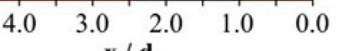

$u=8.0 \mathrm{~m} / \mathrm{s} ; \operatorname{Re}=5.8 \cdot 10^{4}$

Fig. 18. For configuration No. 2 of the simulated-ice-accreted cable model in the moderately turbulent flow: (a) streamline patterns, (b) scalar maps of the instantaneous velocity field in the near-wake, and (c) locations of the flow boundary layer separation point determined at three free stream velocities 3.6, 5.0 and $8.0 \mathrm{~m} / \mathrm{s}$.

the widest vortex street was accompanied by a cross-stream spacing between two free shear layers equal about to $2.0 d(d=12.0 \mathrm{~cm})$ while the distance between two rows of vortices was approximately $1.3 d$. Similarly, the relative increase in vortex size of the primary mature pair of vortices downstream of the model for configuration No. 2 and $2 b$ is almost the same, as shown in Figs. 17 and 19. Since flow-induced forces are coupled with the formation of a vortex street behind the bluff body, this suggests greater vortex-wind forces acting on the ice-accreted bridge cable than in the case of the dry circular cable for a wind direction across the longitudinal axis of a cable-supported bridge, i.e. across the bridge cables. It should be noted that for the bridge cables in cross-flow cable inclination is unimportant. The formation length for configuration No. 2 was within the range of $1.3 d-1.6 d$, while the separation points, as specified using time-averaged flow data, occurred at $69^{\circ}-77^{\circ}$ and $76^{\circ}-92^{\circ}$, at the upper and lower side of the simulated-ice-accreted model, respectively. The mean flow velocity within the sidewise regions was $1.5 u-1.7 u$, while in the near-wake region it was $0.2 u-0.3 u$.

In the case of configuration No. 2 or $2 \mathrm{~b}$ in the moderately turbulent flow conditions (see Figs. 18 and 20), similar to the circular cylinder, weakening of the vortex excitation associated with the increase of free stream turbulence was observed. The vortex street becomes narrower and the sizes of the vortices become obviously smaller. The boundary layer gets separated on the upper and lower sides at a larger mean angle within the range of $94^{\circ}-103^{\circ}$ and $91^{\circ}-98^{\circ}$, respectively. One can assume that the delayed flow separation on both sides of the model surface is due to the early boundary layer transition from laminar to turbulent. In this case the formation length was $0.8 d-1.2 d$, the cross-stream distance between two primary vortices was about $1.0 d$, while the width of the nearwake was about 1.4d-1.5d. The mean flow velocity within the sidewise regions was in the range of $1.1 u-1.2 u$, while in the near-wake region it was $0.1 u-0.3 u$.
In the case of configuration No. 3 in both the low and moderately turbulent flow (see Figs. 21 and 22) a similar near-wake structure, as in the case of the near-wake of the circular cylinder, was found. In the low turbulent flow the mean separation placed at $86^{\circ}-99^{\circ}$ was accompanied by a vortex street width in the range of $0.9 d-1.2 d(d=11.3 \mathrm{~cm})$ and a cross-stream spacing of the shear layer envelope around 1.8d-2.0d. The formation length was approximately $1.2 d-1.5 d$ while the mean flow velocity in the sidewise regions reached $1.5 u$ and $0.4 u-0.6 u$ in the nearwake region.

Contrary to the low turbulent flow, the observed phenomenon of vortex excitation in the moderately turbulent flow was strongly weakened. Clearly repeatable vortex patterns, and specifically a clear repetition of the second vortex, were barely discernible. In spite of this, the following approximate geometric features of the near-wake behind the model were established. The separation was slightly shifted downstream to a mean position in the range of $96^{\circ}-101^{\circ}$. The width of the vortex street decreased to approximately $0.8 d$, and the cross-stream spacing of the shear layers decreased to the range of $1.2 d-1.5 d$. The formation length was between $1.0 d-1.2 d$, while the mean flow velocity in the sidewise regions and the near-wake were approximately $1.1 u-1.3 u$ and $0.2 u$, respectively.

A tabulated summary of the PIV experimental results are given in Table 1.

\section{Concluding remarks}

The focus of this study is PIV analysis of the near-wake character and vortex shedding patterns in the flow past the ice-accreted circular cylinder representing a section model of a bridge cable. The model was analysed in the low and moderately turbulent flow conditions with respect to different angles of wind attack at the Reynolds number values 
a)

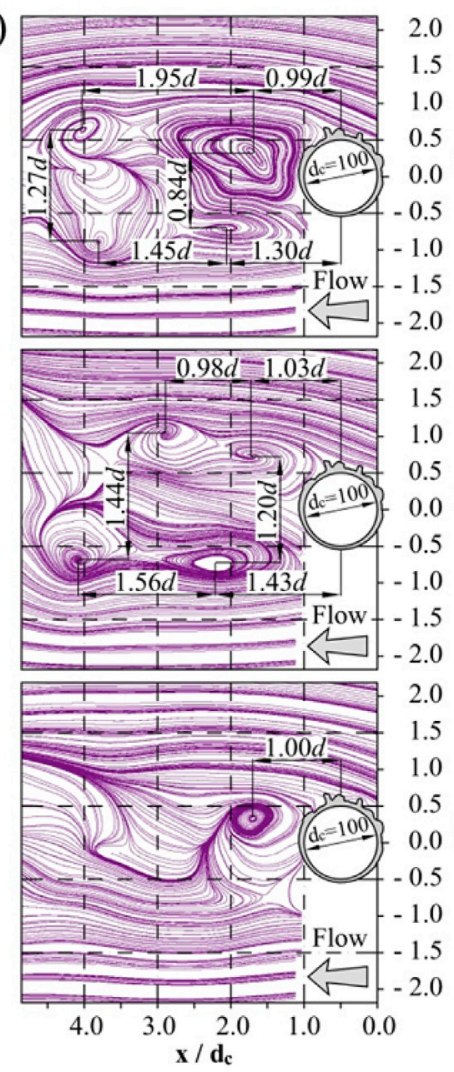

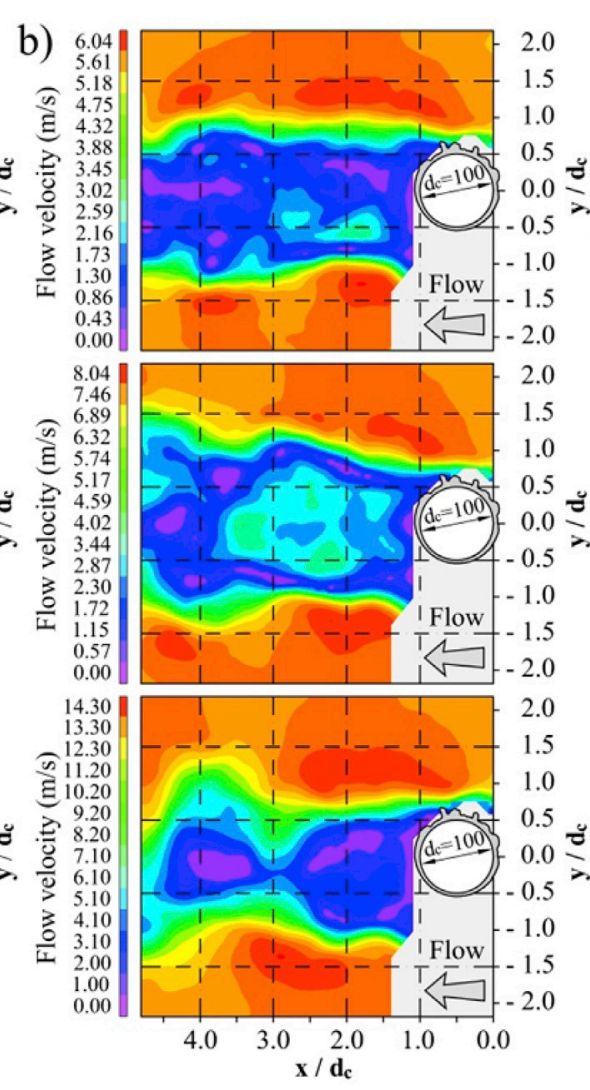

c)

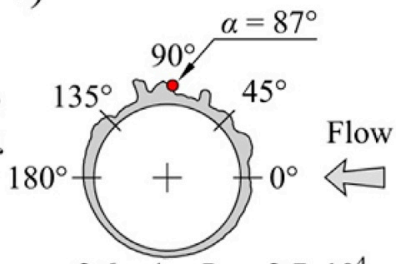

$u=3.6 \mathrm{~m} / \mathrm{s} ; \operatorname{Re}=2.7 \cdot 10^{4}$

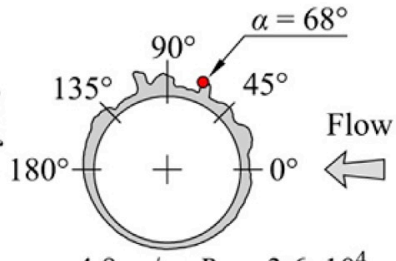

$u=4.9 \mathrm{~m} / \mathrm{s} ; \operatorname{Re}=3.6 \cdot 10^{4}$

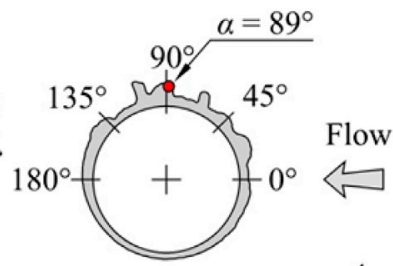

$u=8.7 \mathrm{~m} / \mathrm{s} ; \operatorname{Re}=6.4 \cdot 10^{4}$

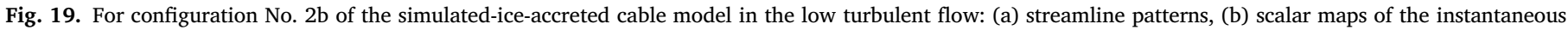
velocity field in the near-wake, and (c) locations of the flow boundary layer separation point determined at three free stream velocities 3.6 , 4.9 and $8.7 \mathrm{~m} / \mathrm{s}$.
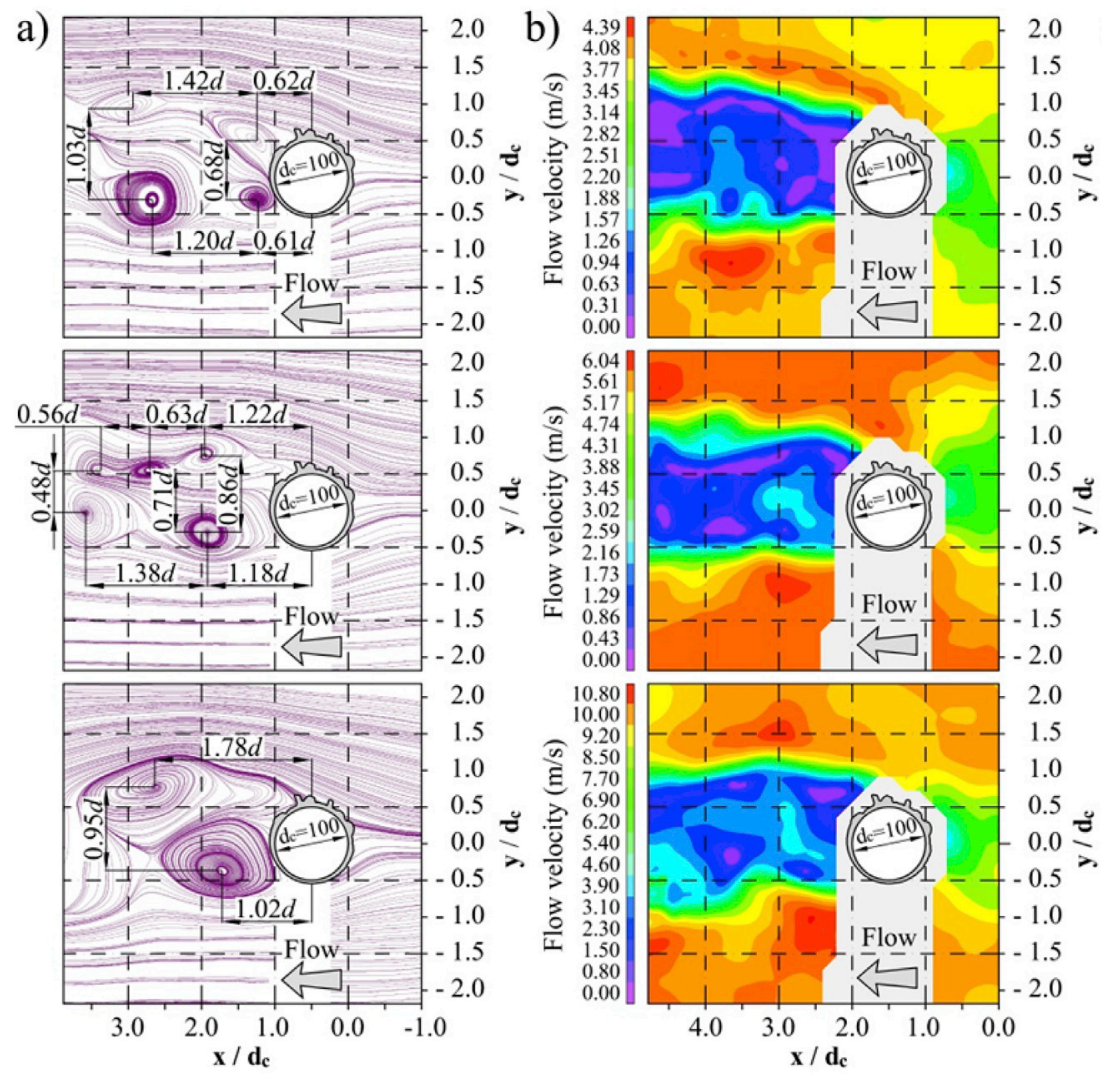

c)
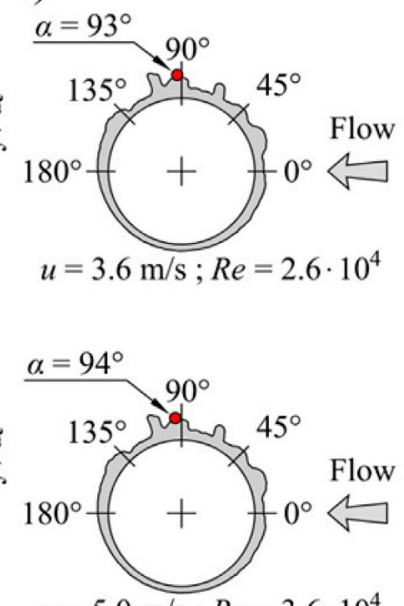

$u=5.0 \mathrm{~m} / \mathrm{s} ; \operatorname{Re}=3.6 \cdot 10^{4}$

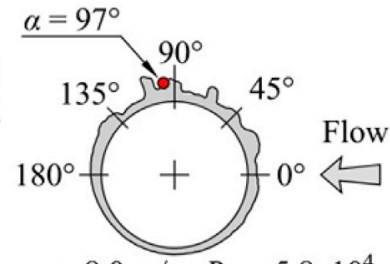

$u=8.0 \mathrm{~m} / \mathrm{s} ; \operatorname{Re}=5.8 \cdot 10^{4}$

Fig. 20. For configuration No. $2 \mathrm{~b}$ of the simulated-ice-accreted cable model in the moderately turbulent flow: (a) streamline patterns, (b) scalar maps of the instantaneous velocity field in the near-wake, and (c) locations of the flow boundary layer separation point determined at three free stream velocities 3.6, 5.0 and $8.0 \mathrm{~m} / \mathrm{s}$. 
a)

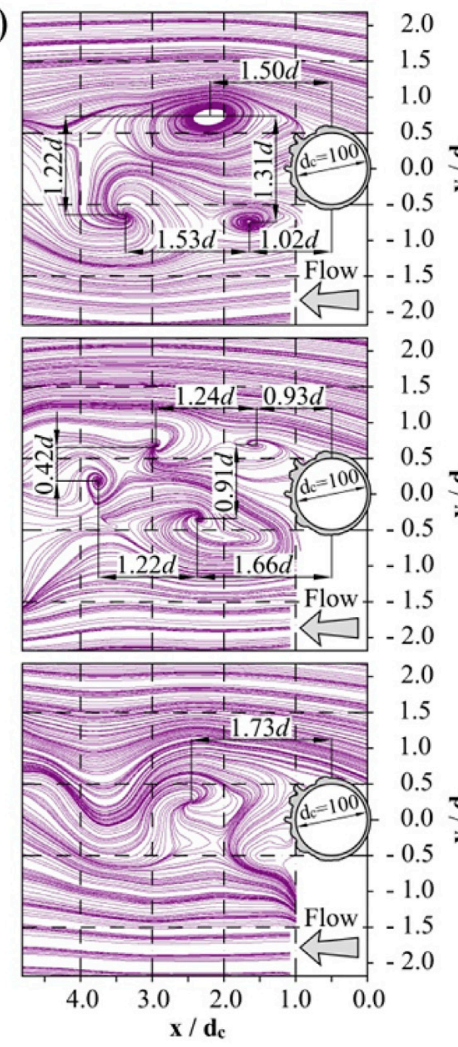

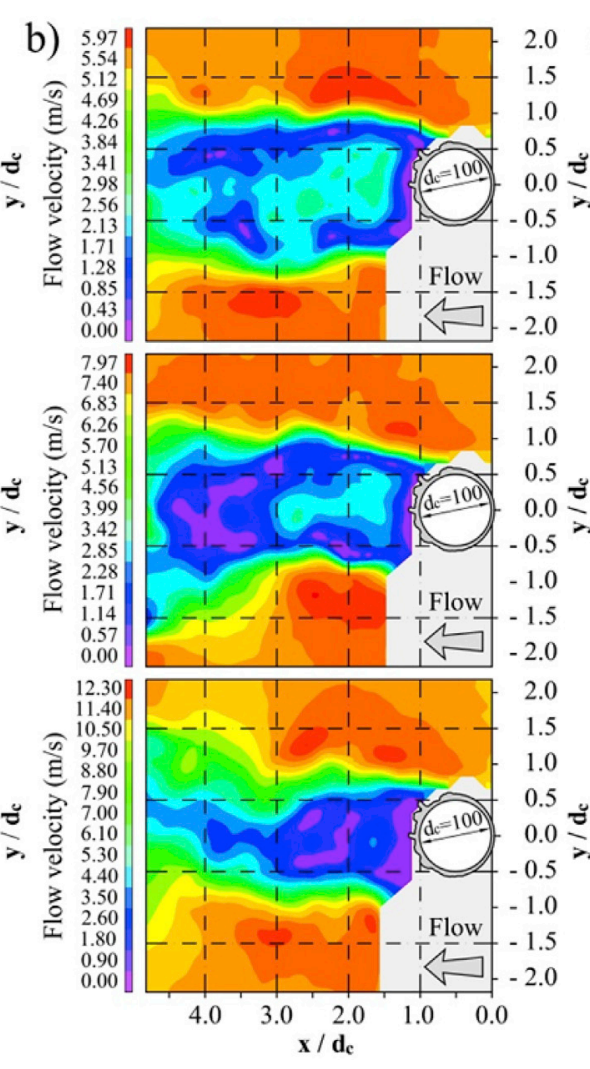

c)

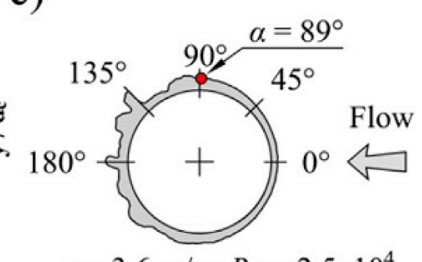

$u=3.6 \mathrm{~m} / \mathrm{s} ; \operatorname{Re}=2.5 \cdot 10^{4}$

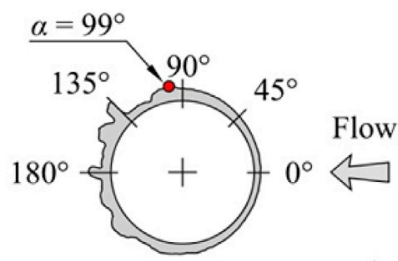

$u=4.9 \mathrm{~m} / \mathrm{s} ; \operatorname{Re}=3.4 \cdot 10^{4}$

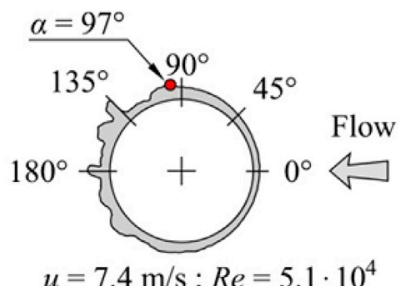

$u=7.4 \mathrm{~m} / \mathrm{s} ; R e=5.1 \cdot 10^{4}$

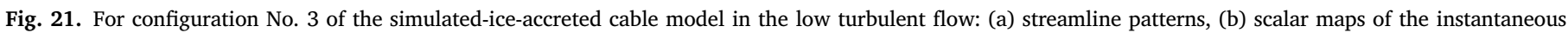
velocity field in the near-wake, and (c) locations of the flow boundary layer separation point determined at three free stream velocities $3.6,4.9$ and $7.4 \mathrm{~m} / \mathrm{s}$.

within the range of $2.2 \cdot 10^{4}-6.4 \cdot 10^{4}$. Wind tunnel experiments essentially consisted of two parts: the simulation of conditions suitable for ice accretion, and flow field analysis in close proximity of the cylinder. The results for the cases of cable model with simulated ice accretion were compared to the reference case of a smooth cylinder. The evidence of wake vortex shedding was examined by means of PIV analysis. In the studied Reynolds number range, the vortices formed at the cable model had both a different size and shape. This depended on the model configuration and the turbulence intensity of the flow.

Comparing the flow structure in the near-wake past the cylinder in the moderately turbulent flow conditions with that obtained in the low turbulent flow, a weakened vortex formation resulting from the free stream turbulence was observed. The characteristic formation length of the vortices, which decreased by about $35 \%$, resulted in a higher Strouhal number value than in the case of the smooth flow. The vortex shedding mode and the vorticity concentration affects the fluctuating component of the aerodynamic force in the cross-wind direction, consequently affecting the dynamic cross-wind response of the cylinder. The separation points of the boundary layer are shifted downstream as the flow turbulence increases. In the moderately turbulent flow conditions, the free shear layers become more unstable than in the low turbulent flow, which results in a much more irregular shape of the shear layers. Consequently, the width of the near-wake decreased by about $30 \%$ compared to the low turbulent flow, certainly leading to a reduction of the mean drag force acting on the cylinder.

In the case of configuration No. 1 in the low turbulent flow, alternating vortices developed on both sides of the model. This formation is characterized by quasi-regular rhythms. The oscillating wakes generated two staggered rows of vortices with opposite rotation behind the model as in the case of the smooth cylinder, however with an approximately $30-40 \%$ narrower vortex street, and a near-wake approximately $20-30 \%$ narrower. The formation length of the vortices was considerably shorter, i.e. more than twice as short as that of the smooth cylinder. In the moderately turbulent flow conditions, two single vortices per shedding cycle on either side of the simulated-ice-accreted model were simultaneously fed into the downstream. These double vortices were not stationary, but they moved downstream. In these conditions separation of the boundary layer took place slightly earlier, reflecting a 15-30\% narrower vortex street and approximately a $10 \%$ narrower near-wake than in the low turbulent flow. The flow did not generate clearly repeatable vortex patterns within the target area.

In the case of configuration No. 2 or $2 b$, distinct vortex pairs, forming alternately on both sides of the model, appear each shedding cycle. A similar phenomenon occurs in the near-wake of the regular cylinder. However, in the case of the low turbulent flow, a $20-30 \%$ wider vortex street and a $30-40 \%$ wider cross-stream spacing between two free shear layers were created in comparison with the case of the moderately turbulent flow or the dry cylinder in the low turbulent flow. As flow-induced forces are coupled with formation of the vortex street behind the bluff body, this suggests a greater vortex-wind forces acting on the ice-accreted bridge cable than in the case of the dry cylinder. In the case of the moderately turbulent flow, a weakening of vortex excitation associated with the increase of free stream turbulence was observed. The vortex street became narrower and the vortices became obviously smaller.

For configuration No. 3, in both the low and moderately turbulent flow, a near-wake structure similar to that of the circular cylinder was observed. In the low turbulent flow, the boundary layer separation was accompanied by a vortex street whose width was within the range of the cylinder diameter. The cross-stream spacing of the shear layer envelope was almost double the cylinder width. The observed phenomenon of vortex excitation in the moderately turbulent flow was strongly weaker than in the case of the low turbulent flow. Repeatable vortex patterns and a clear repetition of the second vortex, were barely evident. In this case, the vortex street and the near-wake were approximately $10-30 \%$ and 20-30\% narrower, respectively, than in the low turbulent flow.

Despite the significant change in the shape of the cable model surface 
a)

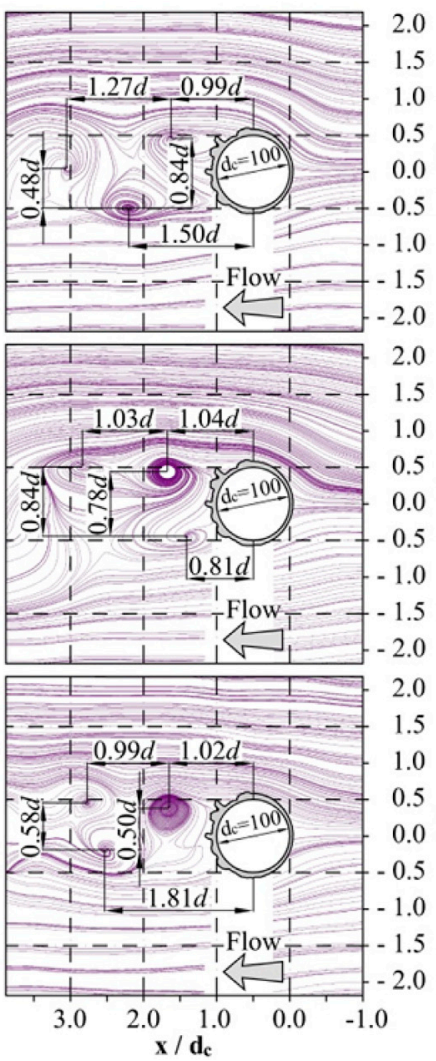

b)
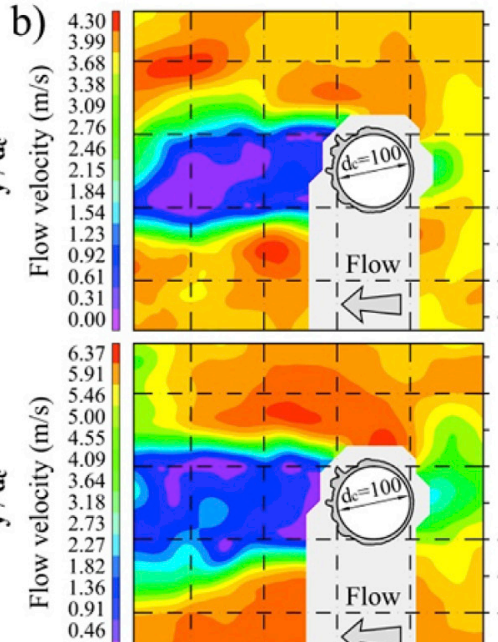

(1)

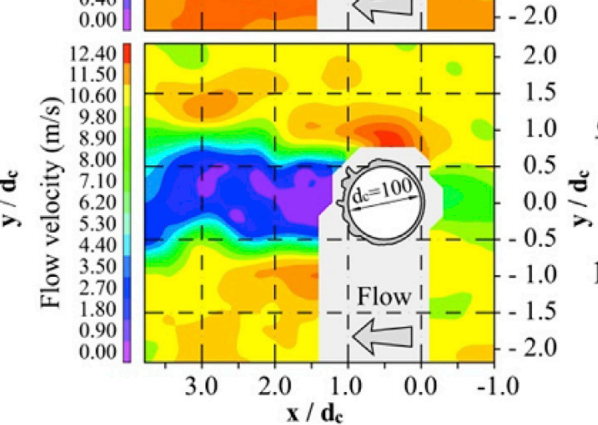

c)

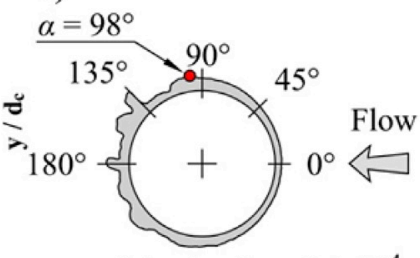

$$
u=3.6 \mathrm{~m} / \mathrm{s} ; \operatorname{Re}=2.4 \cdot 10^{4}
$$

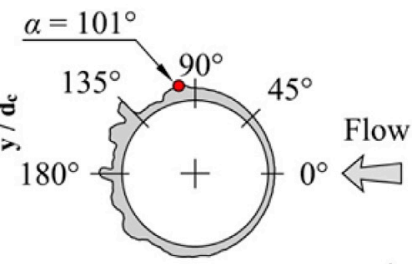

$$
u=5.0 \mathrm{~m} / \mathrm{s} ; R e=3.4 \cdot 10^{4}
$$

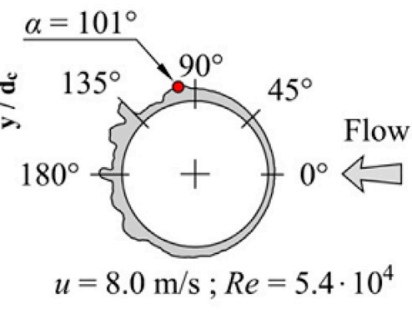

Fig. 22. For configuration No. 3 of the simulated-ice-accreted cable model in the moderately turbulent flow: (a) streamline patterns, (b) scalar maps of the instantaneous velocity field in the near-wake, and (c) locations of the flow boundary layer separation point determined at three free stream velocities 3.6, 5.0 and $8.0 \mathrm{~m} / \mathrm{s}$.

Table 1

\begin{tabular}{|c|c|c|c|c|c|c|c|c|}
\hline \multirow[t]{2}{*}{ Model configuration } & \multirow[t]{2}{*}{ State of the flow } & \multirow[t]{2}{*}{$R e \cdot 10^{4}$} & \multirow[t]{2}{*}{$\begin{array}{l}\text { Mean location of } \\
\text { the separation point }\end{array}$} & \multicolumn{2}{|c|}{$\begin{array}{l}\text { Width in the cross-stream } \\
\text { direction }\end{array}$} & \multirow[t]{2}{*}{$\begin{array}{l}\text { Formation } \\
\text { length }\end{array}$} & \multicolumn{2}{|l|}{ Mean flow velocity } \\
\hline & & & & $\begin{array}{l}\text { of the vortex } \\
\text { street }\end{array}$ & $\begin{array}{l}\text { of the near- } \\
\text { weak }\end{array}$ & & $\begin{array}{l}\text { within the } \\
\text { sidewise regions }\end{array}$ & $\begin{array}{l}\text { within the } \\
\text { near-wake }\end{array}$ \\
\hline \multirow[t]{2}{*}{ Smooth cylinder } & low turbulent & $2.2-5.3$ & $78^{\circ}-80^{\circ}$ & $1.0 d$ & $1.5 d$ & $2.6 d$ & $1.6 u-1.8 u$ & $0.2 u-0.3 u$ \\
\hline & $\begin{array}{l}\text { moderately } \\
\text { turbulent }\end{array}$ & $2.2-4.8$ & $95^{\circ}-102^{\circ}$ & $0.8 d$ & $1.0 d$ & $1.7 d$ & $1.1 u-1.5 u$ & $0.2 u$ \\
\hline \multirow{2}{*}{ Configuration No. 1} & low turbulent & $2.5-6.0$ & $96^{\circ}-101^{\circ}$ & $0.8 d$ & $1.2 d$ & $1.2 d$ & $1.4 u-1.6 u$ & $0.3 u$ \\
\hline & $\begin{array}{l}\text { moderately } \\
\text { turbulent }\end{array}$ & $2.4-5.4$ & $82^{\circ}-102^{\circ}$ & $0.6 d-0.7 d$ & $1.1 d$ & $0.9 d$ & $1.1 u-1.2 u$ & $0.2 u-0.3 u$ \\
\hline \multirow{2}{*}{ Configuration No. 2 (2b) } & low turbulent & $2.7-6.4$ & $69^{\circ}-77^{\circ}\left(76^{\circ}-92^{\circ}\right)$ & $1.3 d$ & $2.0 d$ & $1.3 d-1.6 d$ & $1.5 u-1.7 u$ & $0.2 u-0.3 u$ \\
\hline & $\begin{array}{l}\text { moderately } \\
\text { turbulent }\end{array}$ & $2.6-5.8$ & $94^{\circ}-103^{\circ}\left(91^{\circ}-98^{\circ}\right)$ & $1.0 d$ & $1.4 d-1.5 d$ & $0.8 d-1.2 d$ & $1.1 u-1.2 u$ & $0.1 u-0.3 u$ \\
\hline \multirow[t]{2}{*}{ Configuration No. 3} & low turbulent & $2.5-5.1$ & $86^{\circ}-99^{\circ}$ & $0.9 d-1.2 d$ & $1.8 d-2.0 d$ & $1.2 d-1.5 d$ & $1.5 u$ & $0.4 u-0.6 u$ \\
\hline & $\begin{array}{l}\text { moderately } \\
\text { turbulent }\end{array}$ & $2.4-5.4$ & $96^{\circ}-101^{\circ}$ & $0.8 d$ & $1.2 d-1.5 d$ & $1.0 d-1.2 d$ & $1.1 u-1.3 u$ & $0.2 u$ \\
\hline
\end{tabular}

Summary of the PIV experimental data.

caused by ice accretion, the phenomenon of vortex excitation in the nearwake of the simulated-ice-accreted model was clearly observed having features similar to those of a dry cylinder. Such a statement applies for all tested configurations in both the low and moderately turbulent flow conditions. However, free stream turbulence interacts with the free shear layer and the near-wake, having a significant effect on the vorticity concentration and shedding modes. For this reason, it would be justified to perform further studies aimed at a more complete understanding of the full description of the vortex excitation phenomenon. This would mean expanding experimental study, under various flow conditions, the Strouhal number and the fluctuating component of the aerodynamic lift force acting on ice-accreted cables as a function of the Reynolds number. Since only fundamental research was carried out in the presented study, the authors hope that this work will be an inspiration for further extensive studies on the near-wake flow patterns of ice-accreted bridge cables and the mechanisms of their vibrations. 


\section{Acknowledgments}

This research was supported by the Czech Science Foundation, Czech Republic (grant No. GAČR 19-04695S). The authors would like to dedicate this work to our good friend professor Sergey Kuznetsov, who passed away recently. He was the head of the wind tunnel laboratory of ITAM. In his life he was active in environmental aerodynamics and wind engineering and up to his last moments he maintained an encouraging attitude towards research, working and teaching, which enriched the lives of his students, colleagues and friends.

\section{References}

Adachi, T., 1997. Effects of surface roughness on the universal Strouhal number over the wide Reynolds number range. J. Wind Eng. Ind. Aerodyn. 69-71, 399-412.

Buresti, G., 1981. The effect of surface roughness on the flow regime around circular cylinders. J. Wind Eng. Ind. Aerodyn. 8, 105-114.

Cao, S., Jalali, H.H., Dragomirescu, E., 2018. Wind-induced response of inclined and yawed ice-accreted stay cable models. Shock Vib. 6853047, 1-12, 2018, No.

Cheung, J.C.K., Melbourne, W.H., 1983. Turbulence effects on some aerodynamic parameters of a circular cylinder at supercritical numbers. J. Wind Eng. Ind. Aerodyn. 14 (1-3), 399-410.

Demartino, C., Koss, H., Ricciardelli, F., 2013a. Experimental study of the effect of icing on the aerodynamics of circular cylinders - Part I: cross flow. In: Proceedings of the 6th European and African Wind Engineering Conference. Robinson College, Cambridge, UK.

Demartino, C., Georgakis, C.T., Ricciardelli, F., 2013b. Experimental study of the effect of icing on the aerodynamics of circular cylinders - Part II: inclined flow. In: Proceedings of the 6th European and African Wind Engineering Conference. Robinson College, Cambridge, UK.

Demartino, C., Koss, H.H., Georgakis, C.T., Ricciardelli, F., 2015. Effects of ice accretion on the aerodynamics of bridge cables. J. Wind Eng. Ind. Aerodyn. 138, 98-119.

Demartino, C., Ricciardelli, F., 2015. Aerodynamic stability of ice-accreted bridge cables. J. Fluids Struct. 52, 81-100.

Demartino, C., Ricciardelli, F., 2017. Aerodynamics of nominally circular cylinders: a review of experimental results for Civil Engineering applications. Eng. Struct. 137, 76-114.

Gjelstrup, H., Georgakis, C., Larsen, A., 2007. A preliminary investigation of the hanger vibrations on the Great Belt East Bridge. In: Proceedings of the 7th International Symposium on Cable Dynamics, Vienna, Austria.
Gjelstrup, H., Georgakis, C.T., Larsen, A., 2012. An evaluation of iced bridge hanger vibrations through wind tunnel testing and quasi-steady theory. Wind Struct. 15 (5), 385-407.

Górski, P., Pospíšil, S., Kuznetsov, S., Tatara, M., Marušić, A., 2016. Strouhal number of bridge cables with ice accretion at low flow turbulence. Wind Struct. 22 (2), 253-272.

Koss, H., Gjelstrup, H., Georgakis, C.T., 2012. Experimental study of ice accretion on circular cylinders at moderate low temperatures. J. Wind Eng. Ind. Aerodyn. 104-106, 540-546.

Koss, H., Lund, M.S.M., 2013. Experimental investigation of aerodynamic instability of iced bridge cable sections. In: Proceedings of the 6th European and African Wind Engineering Conference. Robinson College, Cambridge, UK.

Koss, H.H., Henningsen, J.F., Olsen, I., 2013. Influence of icing on bridge cable aerodynamics. In: Proceedings of the 15th International Workshop on Atmospheric Icing of Structures (IWAIS XV), St. John's, Newfoundland and Labrador. Canada.

Kuznetsov, S., Pospíśil, S., Král, R., 2015. Climatic wind tunnel for wind engineering tasks. Technical Transactions 12 (2-B), 303-316.

Li, S., Wu, T., Huang, T., Chen, Z., 2016. Aerodynamic stability of iced stay cables on cable-stayed bridge. Wind Struct. 23 (3), 253-273.

Niemann, H.J., Hölscher, N., 1990. A review of recent experiments on the flow past circular cylinders. J. Wind Eng. Ind. Aerodyn. 33, 197-209.

Nims, D., 2011. Ice Prevention or Removal on the Veteran's Glass City Skyway Cables. Interim Report, Toledo, Spain. State Job Number 134489.

Pantazopoulos, M.S., 1994. Vortex-induced vibration parameters: critical review. In: Proceedings of the 17th International Conference on Offshore Mechanics and Arctic Engineering, Osaka, Japan.

Raffel, M., Willert, C.E., Scarano, F., Kähler, C.J., Wereley, S.T., Kompenhans, J., 2018. Particle Image Velocimetry. A Practical Guide, third ed. Springer International Publishing AG, Cham, Switzerland.

Roldsgaard, J.H., Kiremidjian, A., Georgakis, C.T., Faber, M.H., 2013. Preliminary probabilistic prediction of ice/snow accretion on stay cables based on meteorological variables. In: Proceedings of the 11th International Conference on Structural Safety and Reliability, New York, USA.

Santosuosso, B.J., Klein, G.J., Chauvin, M.R., 2012. Martin Olav Sabo Pedestrian Bridge Cable Diaphragm Plate Fracture Investigation. Final Report, Minneapolis, USA.

Savor, Z., Radic, J., Hrelja, G., 2006. Cable vibrations at Dubrovnik bridge. Bridge Struct. $2(2), 97-106$.

Trush, A., Pospíšil, S., Kuznetsov, S., Kozmar, H., 2017. Wind-tunnel experiments on vortex-induced vibration of rough bridge cables. J. Bridge Eng. 22 (10), 1-8. No. 06017001.

Zdravkovich, M.M., 1997. Flow Around Circular Cylinders, vol. 1. Fundamentals. Oxford University Press, USA.

Zhou, Y., Sun, L., Xie, M., 2018. Wind characteristics at a long-span sea-crossing bridge site based on monitoring data. J. Low Freq. Noise Vib. Act. Control 1-17. https:// doi:10.1177/1461348418762962. 